BNL-HET-99/19

PLY-MS-99-24

\title{
Charges from Dressed Matter: Physics and Renormalisation
}

\author{
EMILI BAGAN" \\ Physics Dept. \\ Brookhaven National Laboratory \\ Upton, NY 11973 \\ $U S A$ \\ Martin LaVElle'], AND David MCMullan \\ School of Mathematics and Statistics \\ The University of Plymouth \\ Plymouth, PL4 8 AA \\ $U K$
}

\begin{abstract}
Gauge theories are characterised by long range interactions. Neglecting these interactions at large times, and identifying the Lagrangian matter fields with the asymptotic physical fields, leads to the infra-red problem. In this paper we study the perturbative applications of a construction of physical charges in QED, where the matter fields are combined with the associated electromagnetic clouds. This has been formally shown, in a companion paper, to include these asymptotic interactions. It is explicitly demonstrated that the on-shell Green's functions and S-matrix elements describing these charged fields have, to all orders in the coupling, the pole structure associated with particle propagation and scattering. We show in detail that the renormalisation procedure may be carried out straightforwardly. It is shown that standard infra-red finite predictions of QED are not altered and it is speculated that the good infra-red properties of our construction may open the way to the calculation of previously uncalculable properties. Finally extensions of this approach to QCD are briefly discussed.
\end{abstract}

\footnotetext{
${ }^{1}$ email: bagan@quark.phy.bnl.gov; permanent address: IFAE, Univ. Autònoma de Barcelona, E-08193 Bellaterra (Barcelona), Spain

${ }^{2}$ email: mlavelle@plymouth.ac.uk

${ }^{3}$ email:dmcmullan@plymouth.ac.uk
} 


\section{Introduction}

The description of charged particles is a central task in quantum field theory. This is obvious for theories such as Quantum Electrodynamics (QED) and the weak interaction since particles with such charges, e.g., the electron and the neutrino, are observed as asymptotic, physical particles in experiments. It is still a challenge even in Quantum Chromodynamics (QCD), however, since how the effective quark structure of hadrons emerges from QCD remains an unsolved problem.

In unbroken gauge theories, characterised by massless gauge bosons, there are long range interactions which, it has long been known [1-3], cannot be neglected even for particles which are a long way from each other. But if the coupling does not vanish, then the gauge transformations are non-trivial and, in particular, the matter fields of QED are not gauge invariant and cannot be identified with physical particles. In previous papers we have shown that by a process of dressing the matter fields with a cloud made out of the vector potentials we can construct fields which are locally gauge invariant. Many such invariant fields can be manufactured. The next task is thus twofold: one must identify which of the gauge invariant fields correspond to physically significant variables and carry out the construction of these fields.

In a previous paper [4] (henceforth I) we argued that a particular set of gauge invariant fields correspond to physical charges in QED. In particular we showed that the slow fall-off of the interaction in QED, which is itself a consequence of the masslessness of the gauge boson, means that this interaction cannot be neglected even for charges which are highly separated from each other and that it is not justified to set the QED coupling to zero at large times in scattering processes. These unjustified assumptions generate the infra-red (IR) problem. We demonstrated that the creation and annihilation operators do create physical (gauge invariant) particles (which even have the expected electromagnetic fields!), but that these operators do not correspond to the asymptotic limits of the Lagrangian matter field. We were able to obtain the gauge invariant fields which do, at the correct points on the mass shell, yield physical particles obeying a free asymptotic dynamics. We further presented an equation with which we could generate these dressed fields corresponding to physical charges. For the purposes of this paper, we may summarise the results of $I$ in one equation: we claimf that the locally gauge invariant field

$$
\psi_{v}(x):=h^{-1}(x) \psi(x)=\mathrm{e}^{-i e \chi(x)} \psi(x),
$$

where

$$
\chi(x)=\frac{\mathcal{G} \cdot A}{\mathcal{G} \cdot \partial}
$$

with $\mathcal{G}^{\mu}=(\eta+v)^{\mu}(\eta-v) \cdot \partial-\partial^{\mu}$, corresponds to a charged particle moving with fourvelocity, $u=\gamma(\eta+v)$ where $\eta$ is the time-like unit vector, $v=(0, \boldsymbol{v})$ is the velocity and $\gamma$ is the usual Lorentz factor, $\gamma=1 / \sqrt{1-\boldsymbol{v}^{2}}$. We should reiterate that we may only interpret these fields as describing physical particles at the on-shell point $p=m \gamma(\eta+v)$.

This paper is dedicated to testing in detail the results of $I$ in the context of perturbative calculations in QED. In particular we will study the infra-red and ultra-violet

\footnotetext{
${ }^{4}$ Note that in this paper we neglect phase effects which play no physical role in QED.
} 
(UV) properties of the dressed fields in (11). It will be shown that the IR divergences associated with going on-shell are indeed removed already at the level of S-matrix elements at all orders in perturbation theory. (Some similar results for scalar QED have been announced elsewhere [5, 6]; here we consider the fermionic theory and give details of our calculations and techniques.) Then the UV structure of these fields will be investigated and it will be shown that the dressed fields have a good UV behaviour.

We note that there is, however, in QCD a general non-perturbative obstruction to this construction of effective quarks and this sets a fundamental limit on the validity of quark models [7].

As well as identifying the above mentioned limit on the construction of observable quarks and constructing solutions for dressed electrons, quarks and gluons (the last two in perturbation theory) which have a specific physical identification, the notable successes of this programme include the demonstration, in QED, that the dressed Green's functions for charge propagation possess a good pole structure [8, 9], that $S$-matrix elements for interactions with dressed asymptotic fields are free of on-shell IR divergences [5] and that the paradigm anti-screening behaviour of QCD can be understood in terms of the interaction between two separately gauge-invariant coloured quarks [10].

The structure of this paper is as follows: after outlining the Feynman rules in Sect. 2, (in particular for interactions with the dressing cloud) we study the IR behaviour of the Green's functions of dressed fields in Sect. 3 to all orders in perturbation theory. Then, in Sect. 4, the UV sector comes under attack: it is shown that the renormalisation of the charged fields, understood here as composite operators, is straightforward and that they do not mix with other operators under renormalisation. Other important properties, such as Ward identities and a demonstration that using physical variables yields the correct result for $g-2$, are also proven here. Finally in Sect. 5 our results are summarised and conclusions are drawn. Various calculational details are given in appendices.

\section{Feynman rules}

In order to perform perturbative calculations, we first need to know the Feynman rules. For the standard propagators and vertices we stick to the conventions used in Bjorken and Drell except for the sign of the electric charge, $e$, which we choose to

be the opposite. In this section we briefly outline the derivation of the new set of Feynman rules for the insertions of the dressed electron, which we then summarise in Fig. 11. We will sometimes refer to these extra vertices, typical of the physical Green's functions, as dressing vertices. We recall from the introduction that our dressed matter $\psi_{v}=\mathrm{e}^{-\mathrm{i} e \chi} \psi$ has an explicit dependence on the coupling constant. We therefore expand the exponential and insert each term, $(-\mathrm{i} e \chi)^{n} / n$ !, of the expansion in the appropriate Green's function (one with a $\bar{\psi}$ field and $n$ photon fields). The $n$ ! is seen to cancel upon contracting the photon fields. According to the standard procedure, we Fourier transform the result and amputate the external propagators. The final results in Fig. I can be read off from the expressions for $\chi$ and $\mathcal{G}_{\mu}$ in (2). Note that the vectors $V_{l}$, 


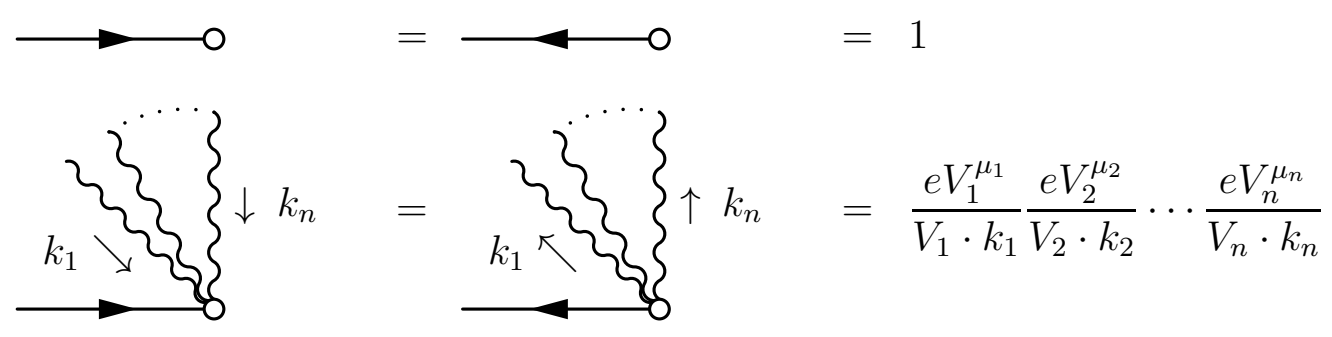

Figure 1: Feynman rules for the insertions of the dressing. We have used the notation $V_{r}^{\mu_{r}}=(\eta+v)^{\mu_{r}}(\eta-v) \cdot k_{r}-k_{r}^{\mu_{r}}$, where $\eta^{\mu}=(1,0)$ and $v^{\mu}=(0, \boldsymbol{v})$. Note that $v^{2}=-\boldsymbol{v}^{2}$.

defined in the caption of Fig. [1, are essentially the Fourier transforms of $\mathcal{G}$ with respect to the variables $k_{l}$. One should also be aware that diagrams such as that in Eq. 81 of Appendix $\mathbb{A}$ have a statistical factor $1 / n$ !, where $n$ is the number of internal photon lines whose ends are both attached to dressing vertices ( $n=2$ in that equation). These lines will be called rainbow lines later on.

\section{Dressed Matter in the Infra-Red}

In this section we wish to study the effects of dressings upon the soft or infra-red structure of gauge theories. IR singularities in gauge theories generally prevent us from following the usual LSZ route to the $S$-matrix, however, in $I$ we argued that it should be possible to pursue the LSZ path using dressed fields. We further recall that in previous papers we have seen that the on-shell dressed matter propagator is free of IR divergences if the dressing is appropriate to the point on the mass shell where we renormalise (e.g., if we renormalise at $p=(m, 0,0,0)$, then the dressing must be that appropriate to a static charge). This has been seen for a general relativistic charge for both the fermionic [5] and scalar theories [8]. We have elsewhere [5] sketched a proof that, in scalar QED, using the appropriate dressings on the various charged external legs, the IR divergences associated with going on shell cancel for both scattering and pair production in dressed Green's functions and the $S$-matrix. Here we will demonstrate that this holds for the fermionic theory and provide the full details which were not given in Ref. [5]. In particular, we will prove the property of factorisation of rainbow lines which was stated without proof in our earlier paper.

We start this section with a study of the one-loop Green's functions of our dressed charges. We will here study the contribution of soft momenta in loop integrals, since only they can generate IR divergences, and, to this purpose, we introduce a refined method with which we can rapidly show the cancellation of on-shell IR divergences. All terms which cannot generate IR problems will be systematically discarded. (This is of course a gauge invariant procedure since our physical charged fields are themselves by construction gauge invariant.) We shall apply this technique to the propagator and also to scattering off a source. It should become clear that this cancellation only holds for charged fields taken at the appropriate points on the mass shell. We then move on to an all orders study. Here we discuss the form of the propagator which tells us about the structure of the renormalisation constants. It is shown that the IR divergences in the 
$S$-matrix elements which describe charged matter scattering off a source円exponentiate and, from our one-loop result, this implies that they are IR finite at all orders in perturbation theory. Since the on-shell renormalisation constants for our dressed fields are IR finite, these results for $S$-matrix elements are then straightforwardly carried over to the dressed Green's functions at the right points on the mass shells. These arguments make extensive use of the factorisation property shown in Appendix A.

\subsection{One-Loop Green's Functions}

To calculate $S$-matrix elements for some general process, we need to calculate on-shell Green's functions and amputate a leg for each external particle. As is well known, the IR problem reveals itself as divergences in the residues of these poles. We want here to calculate dressed Green's functions and show that the dressings generate new terms which exactly cancel the usual IR divergences, i.e., we demonstrate that $S$-matrix elements constructed in terms of dressed charges are IR finite.

Although below we will see that we do not even need to perform the integrals explicitly to see the cancellation of on-shell IR divergences in dressed Green's functions and the corresponding $S$-matrix elements, we should briefly discuss how such divergences may be handled. It is clear that we want to respect gauge invariance and dimensional regularisation is therefore a natural regularisation scheme for us. Here [9] the IR divergences show up as divergences in Feynman parameter integrals which yield poles in $1 /(4-D)$. Another method which may be used is that described in Chapter 13 of [11], here we look for poles in $1 / k^{2}$, use the residue theorem to integrate over $k_{0}$ and so obtain IR singularities as divergences at the lower limit $(\lambda)$ of the integral over $|\boldsymbol{k}|$. A variation on this last scheme keeps the charged matter slightly off shell, $\Delta=p^{2}-m^{2} \neq 0$, which yields logarithms in $\Delta$. This last approach brings out the primarily on-shell nature of the IR problem. The results of all these methods may, however, be straightforwardly translated into each other with the help of the following 'dictionary'

$$
-\log \frac{\Lambda^{2}}{p^{2}-m^{2}} \leftrightarrow \log \frac{\Lambda}{\lambda} \leftrightarrow \int_{0}^{1} d u u^{D-5}=\frac{1}{D-4}
$$

where the form of $\Lambda$ is irrelevant for the singularity. Since, as far as this section is concerned, we can perform all of the necessary manipulations at the level of integrands, we do not need to explicitly perform the integrations - as such we simply write our inetegrals over four-dimensional momenta. All the above schemes, though, may be directly applied.

\section{a) Propagating Charges}

Let us initially consider the simplest Green's function - that describing a single propagating charge. The relevant diagrams are shown in Fig. 2. It is well known that the usual on-shell propagator (the sum of diagrams 2a and 2e) displays a soft divergence. The propagator of the dressed charge, defined as

$$
i S_{v}(p) \equiv\left\langle\psi_{v}(p) \bar{\psi}_{v}(p)\right\rangle \equiv \int \frac{d^{4} x}{(2 \pi)^{4}} \mathrm{e}^{i p \cdot x}\left\langle 0\left|\mathrm{~T} \psi_{v}(x) \bar{\psi}_{v}(0)\right| 0\right\rangle
$$

\footnotetext{
${ }^{5}$ This and the one-loop arguments can be easily generalised to other scattering processes.
} 


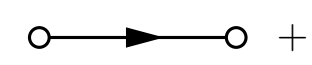

(a)

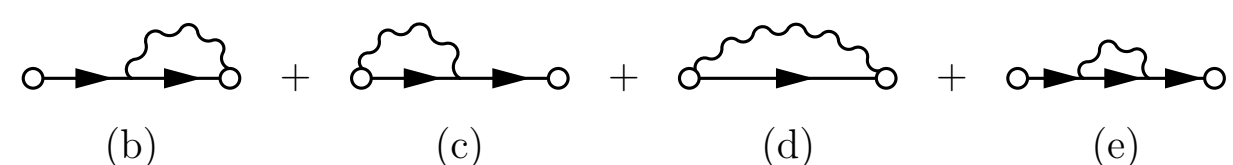

Figure 2: One-loop diagrams contributing to the propagator $i S_{v}(p)$. Note that we display a circle vertex at the end of the fermion lines to show that this is a dressed fermion: at order $g^{0}$ this vertex reduces to unity, but we wish to distinguish it from the usual fermion. This distinction will be more important in the next section.

receives at order $e^{2}$ further corrections from the Feynman rules corresponding to the expansion of the dressings. These terms are represented diagramatically at one loop by the diagrams $2 \mathrm{~b}-\mathrm{d}$.

The contribution of diagram 2 b to the propagator has, from our Feynman rules, the form

$$
i S_{v}^{2 \mathrm{~b}}(p)=-e^{2} \int \frac{d^{4} k}{(2 \pi)^{4}} \frac{V^{\mu}}{V \cdot k} \frac{\not p-\not k+m}{(p-k)^{2}-m^{2}} \gamma^{\nu} D_{\mu \nu}(k) \frac{1}{\not p-m},
$$

where, to help us bring out the gauge invariance of our final result below, we have left the form of the vector boson propagator, $D_{\mu \nu}$, completely general (in Feynman gauge, we would have $\left.D_{\mu \nu}=-g_{\mu \nu} / k^{2}\right)$. The definition of $V$ and its dependence on $v$ should be obvious, cf. Sect. 2. We now drop the term proportional to $k$ in the numerator, as it will be IR finite. We may rewrite $(\not p+m) \gamma^{\nu}$ as $2 p^{\nu}-\gamma^{\nu}(\not p-m)$, and to calculate the on-shell residue of the pole we only have to retain $2 p^{\nu}$. This has then the form

$$
i S_{v}^{2 \mathrm{~b}}(p)=\frac{e^{2}}{\not p-m} \int \frac{d^{4} k}{(2 \pi)^{4}} \frac{V^{\mu} p^{\nu}}{V \cdot k p \cdot k} D_{\mu \nu}(k),
$$

which, we see by power counting, has an IR divergence. The contribution of diagram $2 \mathrm{c}$ is easily seen to be identical to this.

As far as diagram $2 \mathrm{~d}$ is concerned, the Feynman rules yield

$$
i S_{v}^{2 \mathrm{~d}}(p)=e^{2} \int \frac{d^{4} k}{(2 \pi)^{4}} \frac{1}{\not p-\not k-m} \frac{V^{\mu} V^{\nu}}{V \cdot k V \cdot k} D_{\mu \nu}(k) .
$$

In such rainbow diagrams, where one or more vector boson lines extend from one dressing vertex to another, we can, as is shown in Appendix A, so far as the infra-red or ultra-violet structure is concerned, always factor off the loop integrals associated with such lines to obtain a product of the underlying Feynman diagram without the rainbow lines (here the free fermion propagator) times the loop integrals; this is represented diagrammatically in Fig. 3. To do this we make use of Eq. 77 from Appendix A to rewrite this as

$$
i S_{v}^{2 \mathrm{~d}}(p)=\frac{-e^{2}}{\not p-m} \int \frac{d^{4} k}{(2 \pi)^{4}} \frac{V^{\mu} V^{\nu}}{V \cdot k V \cdot k} D_{\mu \nu}(k) .
$$




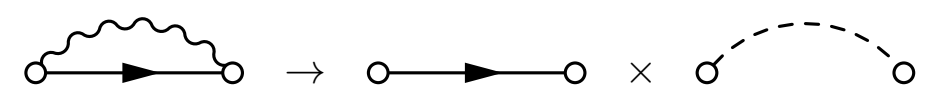

Figure 3: Diagrammatic representation of the factorisation of the one-loop propagator rainbow diagram.

Finally we need to find the contribution of the usual covariant diagram, 2e. From the Feynman rules we obtain

$$
i S_{v}^{2 \mathrm{e}}(p)=\frac{e^{2}}{\not p-m} \int \frac{d^{4} k}{(2 \pi)^{4}} \gamma^{\mu} \frac{1}{\not p+\not k-m} \gamma^{\nu} \frac{1}{\not p-m} D_{\mu \nu}(k),
$$

which appears to have a double pole and to be IR finite. This double pole will be killed off by mass renormalisation. However, we can extract a power of $\left(p^{2}-m^{2}\right)$ and in the process also obtain a single pole with an IR divergence. Proceeding now as with diagram $2 \mathrm{~b}$, we may rewrite this as

$$
i S_{v}^{2 \mathrm{e}}(p)=\frac{e^{2}}{\not p-m} \int \frac{d^{4} k}{(2 \pi)^{4}} \gamma^{\mu} \frac{2 p^{\nu}}{\left[(p-k)^{2}-m^{2}\right]} \frac{1}{\not p-m} D_{\mu \nu}(k),
$$

plus terms with a single pole which are IR finite and hence not considered here. If we expand the integral in $\left(p^{2}-m^{2}\right)$ we obtain

$$
i S_{v}^{2 \mathrm{e}}(p)=-\frac{e^{2}}{\not p-m} \int \frac{d^{4} k}{(2 \pi)^{4}} \gamma^{\mu} \frac{2 p^{\nu}\left(p^{2}-m^{2}\right)}{(2 p \cdot k)^{2}} \frac{1}{\not p-m} D_{\mu \nu}(k) .
$$

where we have also dropped the non-pole like, IR finite terms (i.e., everything except the simple pole). We now write $\left(p^{2}-m^{2}\right)=(\not p+m)(\not p-m)$ : the latter factor here removes the double pole and the first factor can be taken through the remaining gamma matrix to yield effectively $2 p^{\mu}$ (plus terms which do not contain a pole in the propagator). In this way we obtain

$$
i S_{v}^{2 \mathrm{e}}(p)=-\frac{e^{2}}{\not p-m} \int \frac{d^{4} k}{(2 \pi)^{4}} \frac{p^{\mu} p^{\nu}}{(p \cdot k)^{2}} D_{\mu \nu}(k)
$$

We now combine the results for the various diagrams to obtain the following form for the IR divergent terms in the residue of the simple pole in the dressed matter propagator:

$$
i S_{v}^{\mathrm{IR}}(p)=-\frac{e^{2}}{\not p-m} \int \frac{d^{4} k}{(2 \pi)^{4}}\left[\frac{p^{\mu}}{p \cdot k}-\frac{V^{\mu}}{V \cdot k}\right] D_{\mu \nu}(k)\left[\frac{p^{\nu}}{p \cdot k}-\frac{V^{\nu}}{V \cdot k}\right] .
$$

This form confirms the gauge invariance of our construction: any photon propagator other than that of Feynman gauge will involve either a $k_{\mu}$ or a $k_{\nu}$ factor, but these extra terms will vanish on multiplying into the square brackets in (13).

Before showing that this divergence cancels, we should like to introduce our renormalisation conventions. From previous work [9] we know that the wave function renormalisation constant for dressed fermions is in general a matrix. For the scalar theory 
this is naturally not the case, and we stress that for both the scalar [8] and fermionic [9] theories we were able to multiplicatively renormalise the propagator of dressed matter moving at an arbitrary relativistic velocity. We will return to this in Sect. 1 and generalise these results. We define a wave-function matrix renormalisation constant, $\sqrt{\mathbf{Z}_{v}}$, for charged matter moving with four-velocity $\gamma(\eta+v)$ :

$$
\psi_{v}^{\mathrm{B}}=\sqrt{\mathbf{Z}_{v}} \psi_{v}^{\mathrm{R}} \Rightarrow S_{v}^{\mathrm{B}}=\sqrt{\mathbf{Z}_{v}} S_{v}^{\mathrm{R}} \sqrt{\overline{\mathbf{Z}_{v}}}
$$

where $\sqrt{\overline{\mathbf{Z}_{v}}}$ is the adjoint (we stress that the matrix is defined as $\sqrt{\mathbf{Z}_{v}}$ for familiarity and is not to be understood as the square root of some matrix) and $\psi_{v}^{\mathrm{B}}$ and $\psi_{v}^{\mathrm{R}}$ are the bare and renormalised physical charged fields.

From (14) and (13) it follows that the IR contribution to the on-shell wave function renormalisation constant is given by

$$
{\sqrt{\mathbf{Z}_{v}}}^{\mathrm{IR}}=i \frac{e^{2}}{2} \int \frac{d^{4} k}{(2 \pi)^{4}}\left[\frac{p^{\mu}}{p \cdot k}-\frac{V^{\mu}}{V \cdot k}\right] D_{\mu \nu}(k)\left[\frac{p^{\nu}}{p \cdot k}-\frac{V^{\nu}}{V \cdot k}\right],
$$

evaluated at that $p$ corresponding to the correct point on the mass-shell, i.e., $p=$ $m \gamma(\eta+v)$.

That this sum of the different IR divergences actually vanishes completely may be seen either by a brute force calculation or, alternatively, by realising that the term

$$
\frac{V^{\mu}}{V \cdot k}=\frac{(\eta+v)^{\mu}(\eta-v) \cdot k-k^{\mu}}{(k \cdot \eta)^{2}-k^{2}-(k \cdot v)^{2}}
$$

can, for the soft divergences, i.e., the region where $k^{2} \approx 0$, be rewritten as

$$
\frac{V^{\mu}}{V \cdot k}=\frac{(\eta+v)^{\mu}(\eta-v) \cdot k-k^{\mu}}{(k \cdot \eta)^{2}-(k \cdot v)^{2}}
$$

and we may now also drop the $k^{\mu}$ term in the numerator here (cf, the argument for the gauge invariance of Eq. 13). So effectively we obtain

$$
\frac{V^{\mu}}{V \cdot k}=\frac{(\eta+v)^{\mu}(\eta-v) \cdot k}{(k \cdot \eta)^{2}-(k \cdot v)^{2}}=\frac{(\eta+v)^{\mu}}{(\eta+v) \cdot k}=\frac{p^{\mu}}{p \cdot k}
$$

where this last step in the effective replacement, $p^{\mu} / p \cdot k \leftrightarrow V^{\mu} / V \cdot k$, only holds if we renormalise at the appropriate point on the mass shell, i.e., $p=m \gamma(\eta+v)$. In this fashion we may read off the cancellation of the IR divergences in the on-shell propagator at one loop. We stress that if we renormalise at a different point on the mass shell, then the IR divergences are not cancelled. (This argument is closely related to the one used in Sect.'s 3 and 4 of $I$ to show that the distortion factor is cancelled for our dressed matter.)

We further note that (13) is essentially identical to the result [6, 8] obtained for the scalar theory (once $1 /(\not p-m)$ is replaced by $1 /\left(p^{2}-m^{2}\right)$. This brings out the well known spin-independence of the IR divergences in theories with massive charges.

Having thus shown the cancellation of the various IR divergences which occur in the residue of the propagator evaluated in wave-function, on-shell renormalisation, let us move on to a charge being scattered off a source. 


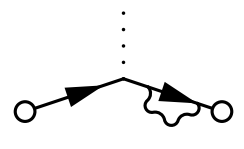

(a)

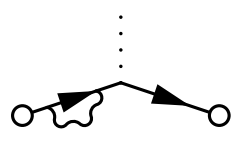

(b)

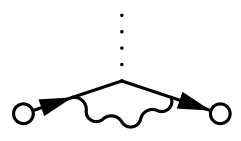

(e)

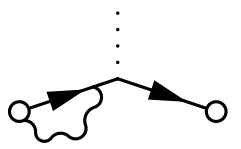

(c)

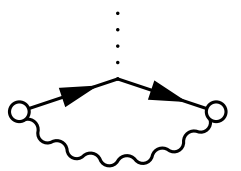

(f)

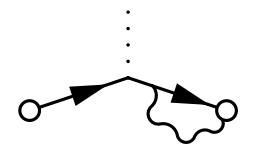

(d)

Figure 4: One-loop diagrams contributing to the scattering of a charge.

\section{b) Scattering Charges}

Consider a charge scattered off a classical source (we denote the vertex generically by $\Gamma)$. At one loop the diagrams are those of Fig. 4 . Most of these diagrams are just propagator corrections on one or other of the legs and as such have been effectively calculated immediately above. The new diagrams are the and 因. We should point out that we here only display those diagrams which can generate an IR divergence and a pole for each of the external charged legs; other figures see, e.g., Fig.'s $9 \mathrm{i}$ and $9 \mathrm{~m}$, will not yield poles in one of the legs:

For the covariant diagram, te, we again take the $\not p+m$ numerator factors through the interaction vertex $\gamma$ matrices and only retain those terms which have a pole for each external leg. In this way we obtain from this diagram

$$
\Gamma^{4 \mathrm{e}}\left(p, p^{\prime}\right)=\frac{i e^{2}}{\not p^{\prime}-m} \Gamma \frac{1}{\not p-m} \int \frac{d^{4} k}{(2 \pi)^{4}} D_{\rho \sigma}(k) \frac{p^{\prime \rho}}{p^{\prime} \cdot k} \frac{p^{\sigma}}{p \cdot k} .
$$

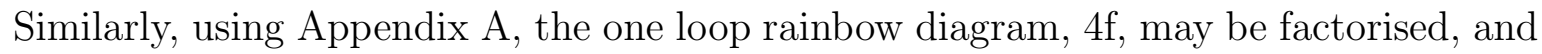
we so find that its contribution to the vertex is

$$
\Gamma^{4 \mathrm{f}}\left(p, p^{\prime}\right)=-\frac{i e^{2}}{\not p^{\prime}-m} \Gamma \frac{1}{\not p-m} \int \frac{d^{4} k}{(2 \pi)^{4}} D_{\rho \sigma}(k) \frac{V^{\prime \rho}}{V^{\prime} \cdot k} \frac{V^{\sigma}}{V \cdot k} .
$$

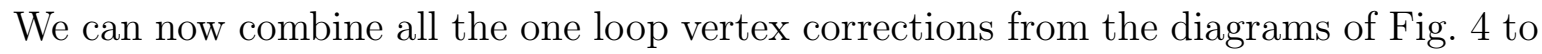
find

$$
\begin{aligned}
\Gamma^{\mathrm{IR}}\left(p, p^{\prime}\right)=\frac{i e^{2}}{\not p^{\prime}-m} \Gamma \frac{1}{\not p-m} & \int \frac{d^{4} k}{(2 \pi)^{4}}\left\{\left[\frac{p^{\rho}}{p \cdot k}-\frac{V^{\prime \rho}}{V^{\prime} \cdot k}\right] D_{\rho \sigma}(k)\left[\frac{V^{\sigma}}{V \cdot k}-\frac{p^{\prime \sigma}}{p^{\prime} \cdot k}\right]\right. \\
& \left.-\left[\frac{p^{\rho}}{p \cdot k}-\frac{p^{\prime \rho}}{p^{\prime} \cdot k}\right] D_{\rho \sigma}(k)\left[\frac{p^{\sigma}}{p \cdot k}-\frac{p^{\prime \sigma}}{p^{\prime} \cdot k}\right]\right\} .
\end{aligned}
$$

\footnotetext{
${ }^{6}$ We should also point out that even if the source is replaced by a photon coupling, as discussed in the next section, then although there are more diagrams at one loop, see Fig. 9, it is still the case that only the diagrams of Fig. \$ have the correct pole structure.
} 
This result again makes the gauge invariant nature of our dressed Green's functions manifest. Furthermore, the on-shell equivalence between $V^{\mu} / V \cdot k$ and $p^{\mu} / p \cdot k$ which we demonstrated above, (18), implies that we can immediately read off that, when both the incoming and the outgoing charge are placed at the correct points on the mass shell, the IR divergences in the on-shell residue cancel. (The spin independence of this structure can again be easily seen by comparing with the results of [5].) It should finally be noted that the dressings associated with the two charges are different for non-zero momentum transfer; there is then no gauge where both dressings vanish and so our general dressed Green's functions cannot be interpreted as ordinary Green's functions in some particular gauge.

These cancellations are a strong indication that our dressings have the physical significance which we argued in I. However, as we will now show, we can greatly improve on these one loop arguments.

\section{$3.2 \quad$ All Orders}

Having seen the cancellation of the IR divergences at leading order, we now want to prove that this cancellation holds to all orders. To do this we will show that the IR divergent terms exponentiate; from this, together with the one loop cancellation, the general statement follows.

\section{a) Propagating Charges}

A general proof of the IR finiteness of the usual matter propagator in the Yennie and radiation gauges was provided many years ago by Jackiw and Soloviev [12 (for the non-covariant gauge they had to use a particular mass-shell renormalisation point.) Essentially these authors established that the (Lagrangian) matter propagators are free of such divergences if a function which they denote by $F_{R}$ vanishes (see their Eq. 3.41). This function is given by

$$
F_{R}=\frac{e^{2}}{(2 \pi)^{3}} \int d^{4} k \mathrm{e}^{i k \cdot x} \theta\left(k_{0}\right) \delta\left(k^{2}\right) \frac{r_{\mu} r_{\nu}}{r^{2}} \Pi^{\mu \nu},
$$

where $r_{\mu}$ is the momentum of the matter, i.e., $m \gamma(\eta+v)$ in our notation, and $\Pi^{\mu \nu}$ is $k^{2}$ times the photon propagator. It is now easy to see that at the correct point on the mass shell our matter propagators will be IR finite to any order, since the dressed propagator is equivalent to the propagator of the Lagrangian matter fields in that gauge where the dressing vanishes. Now in such a dressing gauge the photon propagator is given by

$$
D^{\mu \nu}=\frac{1}{k^{2}} \Pi^{\mu \nu}=\frac{1}{k^{2}}\left[-g^{\mu \nu}+\frac{V^{\mu} V^{\nu}}{V \cdot k}-\frac{V^{2} k^{\mu} k^{\nu}}{(V \cdot k)^{2}}\right],
$$

and so it follows that $u_{\mu} D^{\mu \nu}=0$ in the region, $k^{2}=0$, which is of course enforced by the delta function in (22). Therefore $F_{R}$ vanishes, and the dressed matter propagators are IR finite to any order in perturbation theory, if we renormalise at the appropriate mass shell point, $p=m \gamma(\eta+v)$.

Although this shows that the IR divergences cancel, it is still useful to study the general form of the propagator so as to be able to investigate scattering. 


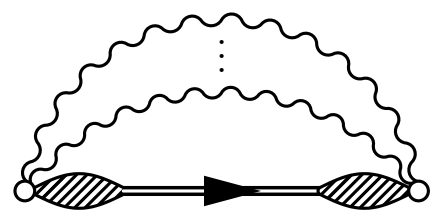

Figure 5: General diagram with rainbow lines for the dressed propagator.

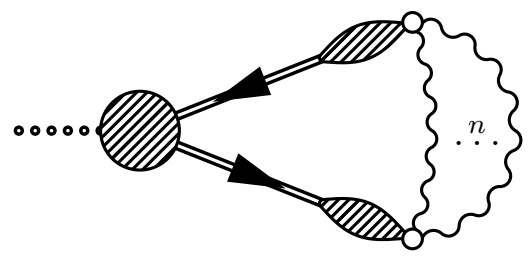

Figure 6: General diagram with rainbow lines for scattering off an external source (the dots).

The general classes of diagrams which can yield IR divergences in the on-shell propagator are shown in Fig. 5. The lines represent the full electron and photon propagators and the blobs correspond to all the diagrams involving the free propagator or also the set of all 1PI diagrams with contributions from the dressing vertex, this we denote algebraically by $G_{v}$ (see also (30) below). Using the factorisation property (see Appendix $\mathrm{A}$ ) we immediately obtain for this figure

$$
G_{v} \frac{i}{\not p-m-\Sigma} G_{v} \mathrm{e}^{-C_{v v}},
$$

where $C_{v v}$ is defined by

$$
C_{v v^{\prime}}=-\int \frac{d^{4} k}{(2 \pi)^{4}} \frac{V \cdot V^{\prime}}{V \cdot k V^{\prime} \cdot k k^{2}} .
$$

Again defining the renormalised propagator as $\left\langle\psi^{B} \bar{\psi}^{B}\right\rangle=\sqrt{\mathbf{Z}_{v}}\left\langle\psi^{R} \bar{\psi}^{R}\right\rangle \sqrt{\overline{\mathbf{Z}_{v}}}$, we find that the wavefunction renormalisation constant has the form

$$
\sqrt{\mathbf{Z}_{v}}=\sqrt{Z_{2}^{\operatorname{cov}}} G_{v} e^{-C_{v v} / 2} .
$$

As discussed above, it follows from the work of Jackiw and Soloviev that this is IR finite at the right point on the mass shell.

\section{b) Scattering Charges}

Consider again a charge scattered off a source. The diagrams which can yield poles and IR divergences are shown in Fig. 6. Using the factorisation property we so obtain

$$
\mathrm{e}^{-C_{v v^{\prime}}} G_{v} \frac{i}{p-m-\Sigma} V_{\text {cov }} \frac{i}{p^{\prime}-m-\Sigma} G_{v^{\prime}},
$$

where $V_{\text {cov }}$ signifies the covariant (dressing independent) central part of these diagrams. To obtain the $S$-matrix element we multiply in with the inverse of the (IR finite) 
renormalisation constants, (26), on each side of the scattering vertex. This kills off the $G_{v}$-type factors. We are left with

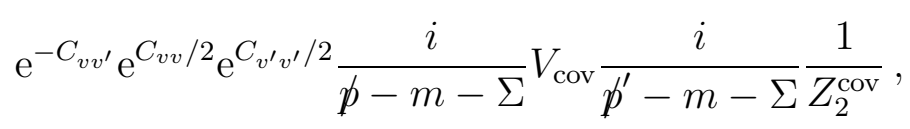

which shows that the dressing contributions to the residues of the two poles exponentiate. As it is well known (see, e.g., Chap. 13 of [11]) that the equivalent dressing independent terms also exponentiate, it follows that all the IR divergent terms in the residues are just the exponentiation of the lowest order result. We have shown that these potential IR divergences cancelled at one loop, hence it follows that this cancellation holds to all orders.

The results of this section provide, we believe, extremely strong evidence for the validity of our formalism. In $I$ we argued that any description of charged matter needs to fulfill two requirements: gauge invariance and also the dressing equation. We were able to solve these conditions to obtain a description of dressed matter and argued that the resulting structures could be identified with the soft and phase structures which characterise the IR problem. In particular, we demonstrated that the asymptotic limit of the fields (1) corresponds to the particle modes (on the right point in the mass shell). All the calculations of this section indicate the correctness of these arguments as we have shown that retaining the soft dressing suffices to remove the soft divergences at all orders in perturbation theory from both the on-shell Green's functions and from $S$-matrix elements where dressed matter is scattered. Now we turn to the UV renormalisation of the non-local and non-covariant fields which describe charged matter.

\section{Renormalisation}

Higher loop effects will, as in any field theory, introduce not only potential IR divergences but also UV singularities which we have to regularise and renormalise. Recall that, to preserve gauge invariance, we regulate the theory by dimensional regularisation. This can after all be directly extended to non-abelian gauge theories. Our notation for the (matrix) wave function renormalisation constants was introduced in the previous section. Here we shall compute operator insertions, propagators and scattering and see where additional renormalisations are required.

As far as our choice of renormalisation scheme is concerned, we will use both onshell and MS schemes. The latter may be most easily generalised to QCD and since we will study composite operator renormalisation, where there is not always a direct physical interpretation, the MS scheme is the most natural. However, there is no loss of generality since the UV divergent parts are the same in all schemes and to obtain the $S$-matrix the finite parts in the various Green's functions can be directly reinstated. 


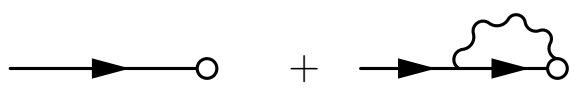

(a)

(b)

Figure 7: Zero- and one-loop 1PI diagrams contributing to the renormalisation of $\psi_{v}$.

\subsection{One Insertion: The Dressed Electron Field as a Composite Operator}

In this section we will investigate the properties of the dressed electron, $\psi_{v}(x)$, understood as a composite operator. In particular we will study its renormalisation at the one-loop level. To this purpose, we shall insert $\psi_{v}(x)$ in Green's functions with an arbitrary number of photon fields. We shall show that these Green's functions can be rendered UV finite by an appropriate multiplicative (matrix) renormalisation of $\psi_{v}(x)$. We will demonstrate that the dressed electron field does not mix under renormalisation with other operators. We would like to stress that, since the physical electron is not a local operator, there is a priori no mathematical guarantee that it can be renormalised. It is therefore highly significant to see that it actually behaves just like a local operator despite its being non-local and non-covariant.

The Feynman rules for the different insertions of the dressing have been obtained in Sect. 2 and are summarised in Fig. 11. Let us start by considering the simplest possible insertion

$$
\left\langle\psi_{v}(p) \bar{\psi}(p)\right\rangle \equiv \int d^{4} x \mathrm{e}^{i p \cdot x}\left\langle 0\left|\mathrm{~T} \psi_{v}(x) \bar{\psi}(0)\right| 0\right\rangle
$$

The corresponding one-particle irreducible $(1 \mathrm{PI})$ part, $G_{v}(p)$, is defined as

$$
\left\langle\psi_{v}(p) \bar{\psi}(p)\right\rangle=G_{v}(p) i S(p)
$$

where $S(p)$ is the standard full electron propagator. Up to one loop, the diagrams contributing to $G_{v}(p)$ are those of Fig. 7 .

One also needs to consider the insertion

$$
\left\langle\psi_{v}(p) \bar{\psi}(p-q) A^{\mu}(q)\right\rangle
$$

The 1PI part of this, $e G_{v \rho}(p, q)$, is now defined through the relation

$$
\left\langle\psi_{v}(p) \bar{\psi}(p-q) A^{\mu}(q)\right\rangle=\left[G_{v}(p) i S(p) i e \Gamma_{\rho}(p, q)+e G_{v \rho}(p, q)\right] i S(p-q) \mathrm{i} D^{\mu \rho}(q),
$$

where $D^{\mu \nu}(q)$ is the full photon propagator and $i e \Gamma_{\rho}(p, q)$ the full vertex, i.e.,

$$
i S(p) i e \Gamma_{\rho}(p, q) i S(p-q) i D^{\mu \rho}(q)=\left\langle\psi(p) \bar{\psi}(p-q) A^{\mu}(q)\right\rangle .
$$

In Fig. 8 we have collected the corresponding 1PI diagrams up to one loop.

Likewise, one can define the 1PI portions of higher insertions. Symbolically we write

$$
e^{n} G_{v \rho_{1} \ldots \rho_{n}}\left(p, q_{1}, \ldots, q_{n}\right)=\left\langle\psi_{v}(p) \bar{\psi}\left(p-\sum q_{i}\right) A_{\rho_{1}}\left(q_{1}\right) A_{\rho_{2}}\left(q_{2}\right) \ldots A_{\rho_{n}}\left(q_{n}\right)\right\rangle^{1 \mathrm{PI}} .
$$




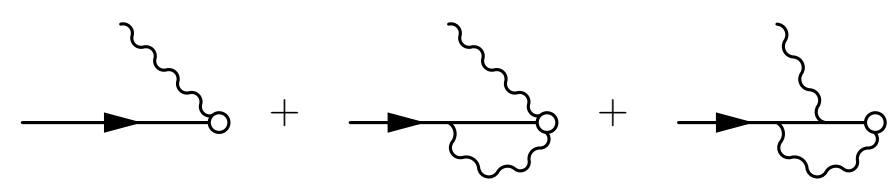

(a)

(b)

(c)

Figure 8: Zero and one loop 1PI diagrams contributing to the renormalisation of $\psi_{v}$.

Eq.'s (30) and (32) are, of course, valid for both the bare and renormalised Green's functions.

Let us now assume that $\psi_{v}(x)$ renormalises multiplicatively. More precisely, let us write

$$
\psi_{v}^{\mathrm{B}}(x)=\sqrt{\mathfrak{z}_{v}} \psi_{v}^{\mathrm{R}}(x) .
$$

This assumption will be correct if we manage to prove that $\sqrt{\mathfrak{z}_{v}}$ suffices to make all $\psi_{v}(x)$ insertions, i.e., Eq. 34, UV finite. It should be emphasised here that $\sqrt{\mathfrak{z}_{v}}$ is not the renormalisation constant introduced in (14). $\sqrt{\mathfrak{z} v}$ renormalises Green's functions with only one insertion of the dressed electron field, whereas $\sqrt{\mathbf{Z}_{v}}$ is introduced to deal with two insertions of the dressed electron field. We will come back to this point below. From (30) and (32) it follows immediately that

$$
\begin{aligned}
G_{v}^{\mathrm{B}}(p) & =Z_{2}^{-1 / 2} \sqrt{\mathfrak{z}_{v}} G_{v}^{\mathrm{R}}(p), \\
G_{v \rho}^{\mathrm{B}}(p, q) & =Z_{2}^{-1 / 2} \sqrt{\mathfrak{z}_{v}} G_{v \rho}^{\mathrm{R}}(p, q),
\end{aligned}
$$

where $Z_{2}$ is the usual electron wave function renormalisation constant. In obtaining (37) we have also used the QED identity $Z_{e}=Z_{3}^{-1 / 2}$, which amounts to charge universality:

$$
e^{\mathrm{B}} A_{\mu}^{\mathrm{B}}(x)=e^{\mathrm{R}} A_{\mu}^{\mathrm{R}}(x) .
$$

Actually, the relation (36) should hold for the 1PI portion of any $\psi_{v}(x)$ insertion.

$$
G_{v \rho_{1} \ldots \rho_{n}}^{\mathrm{B}}\left(p, q_{1}, \ldots, q_{n}\right)=Z_{2}^{-1 / 2} \sqrt{\mathfrak{z} v} G_{v \rho_{1} \ldots \rho_{n}}^{\mathrm{R}}\left(p, q_{1}, \ldots, q_{n}\right) .
$$

This can be seen to be true by noticing that diagram 8c is UV finite by power counting. Therefore,

$$
G_{v \rho}^{\mathrm{B}}(p, q)=G_{v}^{\mathrm{B}}(p) \frac{V_{\rho}}{V \cdot q}+U V \text { finite },
$$

which can be immediately generalised to

$$
G_{v \rho_{1} \ldots \rho_{n}}^{\mathrm{B}}\left(p, q_{1}, \ldots, q_{n}\right)=G_{v}^{\mathrm{B}}(p) \frac{V_{1 \rho_{1}}}{V_{1} \cdot q_{1}} \cdots \frac{V_{n \rho_{n}}}{V_{n} \cdot q_{n}}+U V \text { finite } .
$$

Thus, if $Z_{2}^{-1 / 2} \sqrt{\mathfrak{z}_{v}}$ cancels the UV divergences in Eq. 36, it is automatically guaranteed that $G_{v \rho}^{\mathrm{R}}(p, q), G_{v \rho_{1} \rho_{2}}^{\mathrm{R}}\left(p, q_{1}, q_{2}\right)$, etc., defined through (37) and (39), will also be UV finite.

The calculation of $G_{v}^{\mathrm{B}}(p)$ at one-loop (Fig. $7 \mathrm{~b}$ ) is straightforward. From the Feynman rules we have

$$
G_{v}^{\mathrm{B}(2)}(p)=i e^{2} \int \frac{\mathrm{d}^{2 \omega} k}{(2 \pi)^{2 \omega}} \frac{(\not p-\not k+m)}{\left[(p-k)^{2}-m^{2}\right] k^{2}} \frac{Y}{V \cdot k} .
$$


The UV divergent part solely arises from

$$
\left.G_{v}^{\mathrm{B}(2)}(p)\right|_{\mathrm{div}}=-i e^{2} \int \frac{\mathrm{d}^{2 \omega} k}{(2 \pi)^{2 \omega}} \frac{k^{\alpha} k^{\beta}\left[g_{\alpha \beta}-\gamma_{\alpha}(\not h+\not)(\eta-v)_{\beta}\right]}{\left[(p-k)^{2}-m^{2}\right] k^{2}\left[k^{2}-(k \cdot \eta)^{2}+(k \cdot v)^{2}\right]} .
$$

With the help of Appendix B we obtain

$$
G_{v}^{\mathrm{B}}(p)=1+\frac{e^{2}}{16 \pi^{2}} \frac{1}{\hat{\epsilon}}+\frac{e^{2}}{16 \pi^{2}} \frac{1}{\hat{\epsilon}}\left(\frac{1}{\boldsymbol{v}^{2}}+\frac{1+\boldsymbol{v}^{2}}{2 \boldsymbol{v}^{2}} \chi(\boldsymbol{v})\right) \not h \psi+U V \text { finite. }
$$

We recall now that in Feynman gauge

$$
Z_{2}=1-\frac{e^{2}}{16 \pi^{2}} \frac{1}{\hat{\epsilon}}
$$

and so we finally find

$$
\sqrt{\mathfrak{z}_{v}}=1+\frac{e^{2}}{32 \pi^{2}} \frac{1}{\hat{\epsilon}}+\frac{e^{2}}{16 \pi^{2}} \frac{1}{\hat{\epsilon}}\left(\frac{1}{\boldsymbol{v}^{2}}+\frac{1+\boldsymbol{v}^{2}}{2 \boldsymbol{v}^{2}} \chi(\boldsymbol{v})\right) \not \psi .
$$

Since we have found an explicit solution for Eq. 35 we have demonstrated that our assumption that these operators can be multiplicatively renormalised (and do not mix with other operators) is true. This is a very important result since it would otherwise be a very complicated affair to define a dressing. It is an open question whether this attractive property would hold for gauge invariant dressed fields which do not obey our kinematical requirements on the dressing (see Sect. 3 of $I$ ).

\subsection{Two Insertions}

\subsubsection{The Dressed Propagator}

It is well known (see Chap. 6 of [13]) that a Green's function with several insertions of a renormalised composite operators requires in general further renormalisation. The correlator of two electromagnetic currents is a typical example. In that case (see Sect. 14.6 of [13]), the lowest order diagram is already at one-loop and has an overall divergence that cannot be removed by multiplicative renormalisation. Instead, one has to take derivatives to cancel the UV divergent parts. We shall see that the situation is quite different for the dressed electron field. First of all, the lowest order diagram is just the standard electron propagator (no loops). Hence, there is a possibility that multiplicative renormalisation works. We shall show that this is the case and we shall recognise the extra renormalisation constant to be that of the Isgur-Wise function or, equivalently, that factor which is required to renormalise the cusp UV divergences of Wilson lines. We shall then give an interpretation of this fact.

First, let us consider the correlator $i S_{v}(p)=\left\langle\psi_{v}(p) \bar{\psi}_{v}(p)\right\rangle$. This is the dressed electron propagator already discussed in [9]. Here, we shall recalculate its UV divergent part and see where the different contributions to its renormalisation constant come from. This will give us some insight into the physics of charged matter. The relevant one-loop diagrams are shown in Fig. 2. 
Note that the diagrams $27 \mathrm{a}$ and $2 \mathrm{~b}$ are essentially the same as diagrams $7 \mathrm{a}$ and $7 \mathrm{~b}$ respectively, whereas $2 \mathrm{c}$ is the adjoint of $8 \mathrm{~b}$. All of these diagrams have already been computed in Sect. 4.1. Diagram 2d yields

$$
i S^{2 \mathrm{~d}}(p)=\frac{e^{2}}{\not p-m} \int \frac{\mathrm{d}^{2 \omega} k}{(2 \pi)^{2 \omega}} \frac{V^{2}}{(V \cdot k)^{2}} \frac{\not k(\not p-\not k+m)}{\left[(p-k)^{2}-m^{2}\right] k^{2}} .
$$

As before $V^{\mu}=(\eta+v)^{\mu}(\eta-v) \cdot k-k^{\mu}$, and we have made use of the identity

$$
\frac{1}{\not p-\not k-m}=\frac{1}{\not p-m}\left(1+\not k \frac{1}{p-\not k-m}\right)
$$

and dropped massless tadpole integrals which vanish in dimensional regularisation. The UV divergent part is

$$
i S^{2 \mathrm{~d}}(p)=\frac{-e^{2}}{\not p-m} \int \frac{\mathrm{d}^{2 \omega} k}{(2 \pi)^{2 \omega}} \frac{k_{\mu} k_{\nu}\left[g^{\mu \nu}+\gamma^{-2}(\eta-v)^{\mu}(\eta-v)^{\nu}-2 \eta^{\mu} \eta^{\nu}+2 v^{\mu} v^{\nu}\right]}{\left[(p-k)^{2}-m^{2}\right]\left[k^{2}-(k \cdot \eta)^{2}+(k \cdot v)^{2}\right]^{2}} .
$$

With the help of the integrals collected in Appendix B we get

$$
i S^{2 \mathrm{~d}}(p)=\frac{i}{\not p-m} \frac{e^{2}}{8 \pi^{2}} \frac{1}{\hat{\epsilon}}[1+\chi(\boldsymbol{v})] .
$$

The full result for the UV-divergent part of the propagator up to one loop is thus

$$
\begin{aligned}
i S_{v}^{\mathrm{B}}(p)= & \frac{i}{\not p-m}+G_{v}^{\mathrm{B}(2)}(p) \frac{i}{\not p-m}+\frac{i}{\not p-m} \bar{G}_{v}^{\mathrm{B}(2)}(p)+i S_{v}^{2 \mathrm{~d} \mathrm{~B}}(p) \\
& +\frac{i}{\not p-m}\left[-i \Sigma^{\mathrm{B}(2)}(p)\right] \frac{i}{\not p-m},
\end{aligned}
$$

where $\Sigma^{\mathrm{B}(2)}(p)$ is the usual electron self-energy

$$
\Sigma^{\mathrm{B}(2)}(p)=\frac{3 e^{2}}{16 \pi^{2}} \frac{1}{\hat{\epsilon}} m-\frac{e^{2}}{16 \pi^{2}} \frac{1}{\hat{\epsilon}}(\not p-m) .
$$

Introducing the standard mass shift

$$
m \rightarrow m+\delta m, \quad \frac{\delta m}{m}=\frac{3 e^{2}}{16 \pi^{2}} \frac{1}{\hat{\epsilon}},
$$

Eq. 51 can be written as

$$
\begin{aligned}
i S_{v}^{\mathrm{B}}(p) & =\left\{1+\frac{e^{2}}{16 \pi^{2}} \frac{1}{\hat{\epsilon}}[3+2 \chi(\boldsymbol{v})]\right\} \frac{i}{\not p-m} \\
& +\frac{e^{2}}{16 \pi^{2}} \frac{1}{\hat{\epsilon}}\left[\frac{1}{\boldsymbol{v}^{2}}+\frac{1+\boldsymbol{v}^{2}}{2 \boldsymbol{v}^{2}} \chi(\boldsymbol{v})\right]\left(\not h \psi \frac{i}{\not p-m}-\frac{i}{p-m} \not \phi \psi\right) .
\end{aligned}
$$

We immediately see that the field renormalisation $\sqrt{\mathfrak{z} v}$ introduced in (35) and (46), which would naively imply the possible replacement

$$
S_{v}^{\mathrm{B}}(p)=\sqrt{\mathfrak{z} v} S_{v}^{\mathrm{R}}(p) \sqrt{\mathfrak{\mathfrak { z } v}},
$$


cancels the non-covariant term in (54). However, an extra renormalisation is needed in order to get rid of the first term and one must use the $\sqrt{\mathbf{Z}_{v}}$ renormalisation matrix introduced in (14). If we write $\sqrt{\mathbf{Z}_{v}}=Z_{v}^{1 / 2} \sqrt{\mathfrak{z}_{v}}$, we immediately find

$$
Z_{v}=1+\frac{e^{2}}{8 \pi^{2}} \frac{1}{\hat{\epsilon}}\left[1+\chi\left(\boldsymbol{v}^{2}\right)\right]
$$

as can be read off from Eq. 50.

In [9], we used the following ansatz for the multiplicative renormalisation matrix, $\sqrt{Z_{2}} \exp \left(-\mathrm{i} \sigma_{\mu \nu} \eta^{\mu} v^{\nu} Z^{\prime} / Z_{2}\right)$ for $\sqrt{\mathbf{Z}_{v}}$. Our result in this paper is

$$
\sqrt{\mathbf{Z}_{v}}=1+\frac{e^{2}}{32 \pi^{2}} \frac{1}{\hat{\epsilon}}[3+2 \chi(\boldsymbol{v})]+\frac{e^{2}}{16 \pi^{2}} \frac{1}{\hat{\epsilon}}\left[\frac{1}{\boldsymbol{v}^{2}}+\frac{1+\boldsymbol{v}^{2}}{2 \boldsymbol{v}^{2}} \chi(\boldsymbol{v})\right] \not \eta \psi
$$

which agrees with that of the previous calculation. Note that $\sqrt{\mathbf{Z}_{v}}$ plays the role of $\sqrt{Z_{2}}$ in standard QED. It is the wave function renormalisation constant of the $\psi_{v}(x)$ field.

\subsubsection{The Photon-Dressed Electron Vertex}

The primary source of information on how electrons are scattered by light is the basic correlator $\left\langle\psi_{v^{\prime}}\left(p^{\prime}\right) \bar{\psi}_{v}(p) A_{\mu}(q)\right\rangle$. In this section we shall mainly be concerned with its renormalisation. For bare quantities, let us define the vertex, $i e V_{v v^{\prime}}^{\nu}\left(p, p^{\prime}\right)$, through

$$
i D_{\mu \nu}^{\mathrm{B}}(q) i e^{\mathrm{B}} V_{v v^{\prime}}^{\mathrm{B} \nu}\left(p, p^{\prime}\right)=\left\langle\psi_{v^{\prime}}^{\mathrm{B}}\left(p^{\prime}\right) \bar{\psi}_{v}^{\mathrm{B}}(p) A_{\mu}^{\mathrm{B}}(q)\right\rangle ; \quad q=p^{\prime}-p .
$$

One should not expect that the renormalisation constants introduced so far suffice to make $V_{v v^{\prime}}^{\mu}\left(p, p^{\prime}\right)$ UV finite. As we will see in a moment, a new renormalisation constant, which depends on the relative velocity, is required. The renormalised version of (58) becomes

$$
i D_{\mu \nu}^{\mathrm{R}}(q) i Z\left(v, v^{\prime}\right) e^{\mathrm{R}} V_{v v^{\prime}}^{\mathrm{R} \nu}\left(p, p^{\prime}\right)=\left\langle\psi_{v^{\prime}}^{\mathrm{R}}\left(p^{\prime}\right) \bar{\psi}_{v}^{\mathrm{R}}(p) A_{\mu}^{\mathrm{R}}(q)\right\rangle ; \quad q=p^{\prime}-p
$$

where we have allowed for the above mentioned new renormalisation constant, $Z\left(v, v^{\prime}\right)$. Note that combining Eqs. (58) and (59) we obtain the relation

$$
V_{v v^{\prime}}^{\mathrm{B} \mu}\left(p, p^{\prime}\right)=Z\left(v, v^{\prime}\right) \sqrt{\mathbf{Z}_{v^{\prime}}} V_{v v^{\prime}}^{\mathrm{R} \mu}\left(p, p^{\prime}\right) \sqrt{\overline{\mathbf{Z}_{v}}} .
$$

The need for $Z\left(v, v^{\prime}\right)$ is not that surprising: correlators of renormalised operators, such as that on the right hand side of (59) do not necessarily have to be UV finite. As shall be discussed below, this, and its generalisation to higher Green's functions, will be the last renormalisation constant that we shall need in our approach. It will also become clear in a moment that there is a good physical reason for the introduction of this renormalisation constant.

The correlator $i e V_{v v^{\prime}}^{\mu}\left(p, p^{\prime}\right)$ can be related to the scattering of a physical electron by a classical electromagnetic field. From (59), it is also clear that $Z\left(v, v^{\prime}\right) e^{\mathrm{R}}$ measures the coupling of the dressed electron to a photon. Certainly, at the tree level we reproduce 
the standard result, $i e V_{v v^{\prime}}^{(0)}\left(p, p^{\prime}\right)=i e \gamma^{\mu}$. At zero momentum transfer, we expect that $Z(v, v) e^{\mathrm{R}}$ is the usual charge of the electron, since our claim is that the dressed field $\psi_{v}$ actually describes the experimentally observed electron. Thus, we expect

$$
Z(v, v)=1 .
$$

at all orders. This can actually be proved, as is shown in Appendix Q. This is a very important result, since it ensures that the strength with which photons couple to dressed electrons is given by the standard electric charge $e$, as we claimed at the beginning of this section. One only needs to recall that $e$ is defined at zero momentum transfer, i.e., in the limit $q \rightarrow 0, p^{\prime} \rightarrow p$ and $v^{\prime} \rightarrow v$.

The relevant diagrams for the calculation of the vertex are given in Fig. 9. Note that, for simplicity, we do not include diagrams with vacuum polarisation of the external photon line since they are effectively taken into account by using $e_{\mathrm{R}}$ instead of the bare electric charge $e_{\mathrm{B}}$ in the vertices. In the third and fourth columns of Fig. 9 we collect those diagrams for which the external photon is directly attached to the dressing. For the diagrams in the first two columns, the external photon goes into an interaction vertex. Diagrams $9 \mathrm{i}$ and $9 \mathrm{~m}$ are finite by power counting. Diagrams 9a, 9d, 9e and 9h are standard QED diagrams, i.e., they do not receive contributions from the dressings. It is well known that 9d (or 9e) and 9h cancel each other (once the electron mass has been renormalised)

$$
- \text { [Fig. 9h] }=\text { [Fig. 9d }]=[\text { Fig. 9e }]=\frac{i}{\not p^{\prime}-m} \gamma^{\mu} \frac{i}{\not p-m}\left(-\frac{e^{2}}{16 \pi^{2}} \frac{1}{\hat{\epsilon}}\right) .
$$

This follows from the standard QED Ward identity $Z_{2}=Z_{1}$. Hence, effectively, the first column can be dropped. All the other diagrams can be easily computed from the results above except for 9q, 9r and 9s. The calculation of these last three diagrams can be greatly simplified by choosing the Breit frame where $\boldsymbol{v}^{\prime}=-\boldsymbol{v}$. In this frame we have

$$
\text { [Fig. 9r] }=\frac{i}{\not p^{\prime}-m} \frac{-e V^{\mu}}{V \cdot q} e \int \frac{\mathrm{d}^{2 \omega} k}{(2 \pi)^{2 \omega}} \frac{k_{\nu} k_{\rho}\left[g^{\nu \rho}-\gamma^{-2}\left(\eta^{\nu} \eta^{\rho}-v^{\nu} v^{\rho}\right)\right]}{\left[\left(p^{\prime}-k\right)^{2}-m^{2}\right]\left[k^{2}-(k \cdot \eta)^{2}+(k \cdot v)^{2}\right]^{2}} .
$$

Using the results of Appendix B we finally obtain

$$
\text { [Fig. 9r] }=\frac{i}{\not p^{\prime}-m} \frac{i V^{\mu}}{V \cdot q} \frac{e^{2}}{16 \pi^{2}} \frac{1}{\hat{\epsilon}}\left(1-\boldsymbol{v}^{2}\right) \chi(\boldsymbol{v}) \text {. }
$$

Similarly

$$
\text { [Fig. 9s] }=\frac{-i V^{\prime \mu}}{V^{\prime} \cdot q} \frac{i}{\not p-m} \frac{e^{2}}{16 \pi^{2}} \frac{1}{\hat{\epsilon}}\left(1-\boldsymbol{v}^{2}\right) \chi(\boldsymbol{v}),
$$

where now $V^{\prime \mu}=\left(\eta+v^{\prime}\right)^{\mu}\left(\eta-v^{\prime}\right) \cdot q-q^{\mu}=(\eta-v)^{\mu}(\eta+v) \cdot q-q^{\mu}$.

The calculation of diagram 9q might seem to be much more involved. However, since we are interested just in the UV divergent part, which is easily seen to be independent of the external momenta $p, p^{\prime}$ and $q$, we can set $p^{\prime}=0$ to simplify the loop integral. We end up with the same integral as in (63). The final result is

$$
\text { [Fig. 9q] }=\frac{i}{\not p^{\prime}-m} \gamma^{\mu} \frac{i}{\not p-m} \frac{e^{2}}{16 \pi^{2}} \frac{1}{\hat{\epsilon}}\left(1-\boldsymbol{v}^{2}\right) \chi(\boldsymbol{v}) \text {. }
$$




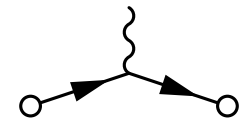

(a)

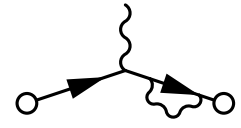

(d)

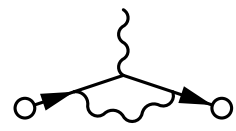

(h)

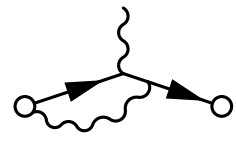

(i)

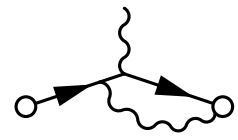

(m)

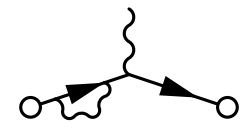

(e)

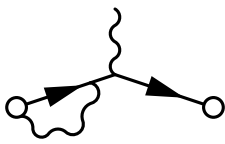

(j)

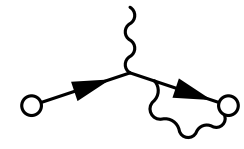

(n)

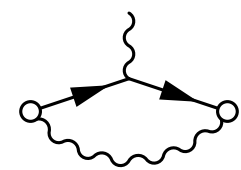

(q)

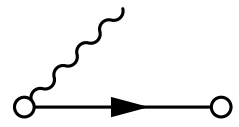

(b)

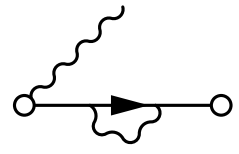

(f)

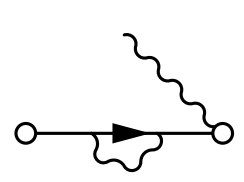

(g)

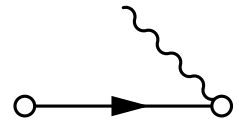

(c)

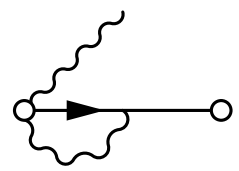

(k)

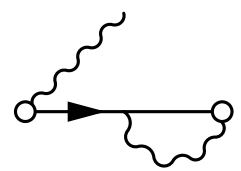

(o)

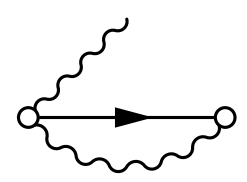

(r)

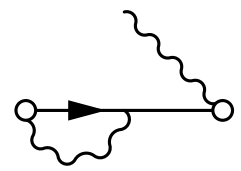

(l)

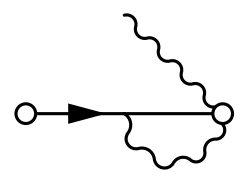

(p)

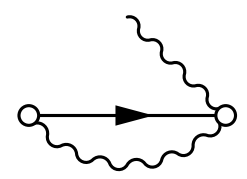

(s)

Figure 9: Zero- and one-loop 1PI diagrams contributing to $V_{v v^{\prime}}^{\mu}\left(p, p^{\prime}\right)$. 
Note that the sum of diagrams 9 $\mathrm{q}, 9 \mathrm{r}$ and $9 \mathrm{~s}$ is just the tree level result (diagrams 9a, 9b and 9c) times the UV divergent factor present in the three equations (64), (65) and (66). Combining these with the results for the other diagrams we have

$$
\begin{aligned}
V_{v v^{\prime}}^{\mathrm{B} \mu}\left(p, p^{\prime}\right) & =V_{v v^{\prime}}^{(0) \mu}\left(p, p^{\prime}\right)\left\{1+\frac{e^{2}}{16 \pi^{2}} \frac{1}{\hat{\epsilon}}\left(1-\boldsymbol{v}^{2}\right) \chi(\boldsymbol{v})\right\} \\
& +\frac{e^{2}}{16 \pi^{2}} \frac{1}{\hat{\epsilon}}\left[\frac{1}{\boldsymbol{v}^{2}}+\frac{1+\boldsymbol{v}^{2}}{2 \boldsymbol{v}^{2}} \chi(\boldsymbol{v})\right]\left(\not h V_{v v^{\prime}}^{(0) \mu}\left(p, p^{\prime}\right)-V_{v v^{\prime}}^{(0) \mu}\left(p, p^{\prime}\right) \eta \psi\right),
\end{aligned}
$$

where $V_{v v^{\prime}}^{(0) \mu}\left(p, p^{\prime}\right)$ is the tree level correlator (the top row of Fig. 9)

$$
V_{v v^{\prime}}^{(0) \mu}\left(p, p^{\prime}\right)=\frac{i}{\not p^{\prime}-m} \gamma^{\mu} \frac{i}{\not p-m}+\frac{i}{p^{\prime}-m} \frac{i V^{\mu}}{V \cdot q}+\frac{-i V^{\prime \mu}}{V^{\prime} \cdot q} \frac{i}{\not p-m} .
$$

From this we can read off that the non-covariant structures do not require any further renormalisation. Taking $(50)$ and $(56)$ into account we find that the extra renormalisation which is needed has the form

$$
Z\left(v, v^{\prime}\right)=1-\frac{e^{2}}{16 \pi^{2}} \frac{1}{\hat{\epsilon}}\left[\left(1+\boldsymbol{v}^{2}\right) \chi(\boldsymbol{v})+2\right] .
$$

To interpret this result, let us introduce $y=u \cdot u^{\prime}$, where $u\left(u^{\prime}\right)$ is the four velocity associated with $\boldsymbol{v}\left(\boldsymbol{v}^{\prime}\right)$. Then, in the Breit frame

$$
y=\frac{1+\boldsymbol{v}^{2}}{1-\boldsymbol{v}^{2}} \Rightarrow \log \left(y+\sqrt{y^{2}-1}\right)=-|\boldsymbol{v}| \chi(\boldsymbol{v})
$$

so that

$$
Z\left(v, v^{\prime}\right)=1+\frac{e^{2}}{16 \pi^{2}} \frac{1}{\hat{\epsilon}}\left[\frac{2 y}{\sqrt{y^{2}-1}} \log \left(y+\sqrt{y^{2}-1}\right)-2\right] .
$$

The corresponding anomalous dimension is

$$
\gamma\left(v, v^{\prime}\right)=\mu \frac{\mathrm{d}}{\mathrm{d} \mu} \log Z\left(v, v^{\prime}\right)=-\frac{e^{2}}{4 \pi^{2}}\left[\frac{2 y}{\sqrt{y^{2}-1}} \log \left(y+\sqrt{y^{2}-1}\right)-2\right],
$$

which we recognise as the anomalous dimension of the Isgur-Wise function [14, 15. This is also the anomalous dimension of the cusp divergences of Wilson loops. We will return to the interpretation of this in a moment.

In the Breit frame, the limit of zero-momentum transfer corresponds of course to $\boldsymbol{v} \rightarrow 0$. It is easy to see that the term in the square brackets in (69) vanishes in this limit and that our Ward identity (61) is indeed fulfilled.

As a final check, one can also compute $Z\left(v, v^{\prime}\right)$ in the rest frame of the incoming particle, namely: $\boldsymbol{v} \rightarrow 0, \boldsymbol{v} \rightarrow \boldsymbol{v}^{\prime}$. In this case, the integral in (63) becomes

$$
\int \frac{\mathrm{d}^{2 \omega} k}{(2 \pi)^{2 \omega}} \frac{\left.k_{\nu} k_{\rho}\left[g^{\nu \rho}-\eta^{\nu} v^{\rho}-\eta^{\nu} \eta^{\rho}+v^{\nu} v^{\rho}\right)\right]}{\left[\left(p^{\prime}-k\right)^{2}-m^{2}\right]\left[k^{2}-(k \cdot \eta)^{2}\right]\left[k^{2}-(k \cdot \eta)^{2}+(k \cdot v)^{2}\right]}=-\frac{1}{8 \pi^{2}} \frac{1}{\hat{\epsilon}},
$$

where we have used Appendix B. The final result is

$$
Z\left(v, v^{\prime}\right)=1-\frac{e^{2}}{16 \pi^{2}} \frac{1}{\hat{\epsilon}}[2+\chi(\boldsymbol{v})] .
$$


Recalling that in this frame $y=1 / \sqrt{1-\boldsymbol{v}^{2}}$ we once more obtain $(71)$.

\section{Physical interpretation}

Smooth Wilson loops are known to be UV finite if the electric charge is renormalised in the usual wayl. If two smooth parts of a loop meet at a point to form a non-zero angle $y$, extra UV divergences arise [17]. These are the so called cusp singularities or divergences. They result from the photon exchange between the two bit of the loop near the singular point or cusp. Physically, the divergence signals the infinite bremsstrahlung produced by the infinite acceleration at a cusp where the heavy charge, which is being driven along the loop, suffers a sudden change of direction. It is well known that these UV cusp divergences require further renormalisation. One needs to introduce a new renormalisation constant which must depend on the angle of the cusp, $Z(y)$.

In the Heavy Quark Effective Theory (HQET) the same phenomenon takes place when a heavy quark suddenly changes its velocity, e.g., after it is 'kicked' by a weak current. This is what happens in, for example, the decay $b \rightarrow c+\ell+\nu_{\ell}$, since, from the point of view of the HQET, the two quarks $b$ and $c$ are identical; both are infinitely heavy quarks; and it is just the quarks velocity that changes. Heavy quark (meson) to heavy quark (meson) transitions are determined by a universal form factor known as the Isgur-Wise function. This encodes the dynamics of the light degrees of freedom that surrounds the heavy quark in a heavy hadron. From our comments above, it follows that, in HQET, $Z(y)$ must renormalise the Isgur-Wise function. This is, again, a standard result in HQET.

For our dressed charges, we are faced with the same situation. The dressing provides a photonic cloud (just like the light degrees of freedom in HQET) which accompanies the charge in its journey to the interaction region. This cloud is such that it provides the right electromagnetic field for the charged particle: that of a charge moving with constant (asymptotic) velocity, $\boldsymbol{v}$. In the remote future, the charges carries with it a cloud appropriate to a different velocity, $\boldsymbol{v}^{\prime}$. From the point of view of the photonic cloud the charge is infinitely heavy or classical (the cloud cannot change the particle's velocity). As far as the dressing is concerned, the scattering is merely an abrupt change of velocity at the point where the two (straight) worldlines characterised by $u=\gamma(1, \boldsymbol{v})$ and $u^{\prime}=\gamma\left(1, \boldsymbol{v}^{\prime}\right)$ intersect. As a result the same infinite bremsstrahlung takes place and an extra renormalisation constant $Z(y)$ is necessary.

\section{$g-2$ for the dressed electron}

The next obvious one-loop test is the calculation of the anomalous magnetic moment of the dressed electron, i.e., $g-2$. We will show that this quantity has the standard QED value, $e^{2} / 4 \pi^{2}$, thus reinforcing our interpretation of the dressed electron as being the electron we actually observe in the laboratory. We need to compute the $S$-matrix element associated to $V_{v v^{\prime}}^{\mu}\left(p, p^{\prime}\right)$ and it is, therefore, convenient to work in the on-shell renormalisation scheme. We recall that the relevant diagrams are in Fig. 9. Actually,

\footnotetext{
${ }^{7}$ There is also a linear divergence proportional to the length of the loop that can be absorbed by an appropriate renormalisation of the (infinite) mass of the charged that it is driven along the loop. This UV divergence does not show up in dimensional regularisation [16].
} 
only those in the first two columns give a non-zero contribution to the $S$-matrix element, thus we can write the $S$-matrix element as

$$
\bar{u}\left(p, s^{\prime}\right) i e \mathfrak{M}_{v v^{\prime}}^{\mathrm{R} \mu}\left(p, p^{\prime}\right) u(p, s)
$$

where the electron momenta are on-shell, i.e., $p=m \gamma(\eta+v), p^{\prime}=m \gamma^{\prime}\left(\eta+v^{\prime}\right)$. Here $\mathfrak{M}_{v v^{\prime}}^{\mathrm{R} \mu}\left(p, p^{\prime}\right)$ denotes the renormalised diagrams that we referred to above after a tree level propagator has been amputated from each of the two fermion legs. A more precise definition of $\mathfrak{M}_{v v^{\prime}}^{\mathrm{R} \mu}\left(p, p^{\prime}\right)$ can be found in App. ․ Note that diagrams $9 \mathrm{i}$ and $9 \mathrm{~m}$ give no contribution to (75) because they have only a single pole in the external electron momenta.

In the on-shell scheme, the self energy-like diagrams 9d, 9 e, 9j, 9n, do not have to be included since $\mathfrak{M}_{v v^{\prime}}^{\mathrm{R} \mu}\left(p, p^{\prime}\right)$ in (75) is a renormalised Green function on the mass shell. This can be most easily seen by switching to the counterterm language for a moment and then noticing that for each of these diagrams there is a counterterm diagram which exactly cancels it on the mass shell. This is in particular true for these four diagrams. The cancellation is complete because we demand the renormalised full propagator of the dressed electron to be the tree level one near the mass shell. One may still worry that this cancellation does not necessarily have to take place diagram by diagram but only for their sum. This is actually the case in general. However, for the argument we present below, it suffices to show that the counterterm of diagrams 9j and 9n exactly cancel against the corresponding loop diagrams, so that one can effectively drop them completely in (75). The counterterm of the loop in $9 \mathrm{j}$ is defined to be the value of the loop in diagram $2 \mathrm{c}$ but with the opposite sign because none of the other diagrams in Fig. 2 are proportional to the structure $[\not p-m]^{-1} \not h \psi\left(\not h \psi^{\prime}\left[\not p^{\prime}-m\right]^{-1}\right)$ - the expression corresponding to diagram $2 \mathrm{~d}$ on the mass shell is given in (8) and is seen to be proportional to the tree level fermion propagator comparing $9 \mathrm{j}(9 \mathrm{n})$ with 2 $2 \mathrm{c}(2 \mathrm{~b})$ it is apparent that the loop integrals are the same and hence the former diagram is completely cancelled by its counterterm, as we claimed. (This argument can be directly extended to diagram 9n but now comparing with $2 \mathrm{~b}$ and making the substitutions $v \rightarrow v^{\prime}$ and $p \rightarrow p^{\prime}$.)

From the previous paragraph it should now be clear that the only non-standard contribution to $g-2$ can possibly come from diagram 9q. It is, therefore, our task to compute the coefficient $\delta F_{2}$ of the magnetic moment structure $i \sigma^{\mu \nu}\left(p^{\prime}-p\right)_{\nu} /(2 m)$ for this diagram because $g-2$ is given in terms of it by

$$
g-2=\frac{e^{2}}{4 \pi^{2}}+2 \delta F_{2}
$$

\footnotetext{
${ }^{8}$ In dimensional regularisation this diagram is zero because (8) is a massless tadpole integral. This diagram is actually both IR and UV divergent and the two $D-4$ poles cancel each other because IR and UV divergences are treated in the same way in dimensional regularisation. If we were regularizing IR divergences by keeping the electron slightly off shell [see comments around Eq. (3)] then the integrand of (8) would have an extra factor

$$
\frac{2 p \cdot k-k^{2}}{2 p \cdot k-k^{2}+\Delta}
$$
}


The expression for this diagram has already been given in (20) wheref one just has to substitute $\gamma_{\mu}$ for $\Gamma$. But then again we see that this diagram is proportional to $\gamma_{\mu}$ (once the external propagators have been amputated) and can contribute only to the form factor widely known as $F_{1}$. Hence, $\delta F_{2}=0$ and $g-2$ has its standard QED value.

\section{Conclusions}

Physical variables in gauge theories have to fulfil Gauss' law, i.e., they must be locally gauge invariant. Since the coupling in the presence of massless gauge bosons does not effectively 'switch off' at very large times, Gauss' law continues to impose a non-trivial constraint on the matter fields. This directly implies that the fundamental matter fields in the original Lagrangian of QED should not be identified with the asymptotic physical fields. In particular all charged fields whether quarks, gluons or electrons must always carry with them a chromo- or electromagnetic cloud and only these systems the matter and its associated dressing cloud taken together - can have any physical meaning.

Of course there are many ways to produce such gauge invariant constructs and the first task is to discover which descriptions have any physical relevance. To this aim we required in $I$ two things of any description of a physical particle: i) local gauge invariance; and ii) that the dressing obeys an equation which depends upon the velocity of the charged particle. In QED we were able to solve these requirements and so found a general description of charged particles. These gauge invariant, dressed fields were shown to have a precise physical interpretation in the asymptotic region: their modes, taken at the appropriate point on the mass shell, are free particle modes. The distortion factor which prevents any particle identification of the matter fields in relativistic QED [3] was demonstrated to be cancelled by the dressing, which is strong evidence that our dressings indeed incorporate the asymptotic dynamics.

In this paper we have further tested these physical variables in a wide variety of field theoretical calculations. Since any description of a physical charged particle is necessarily both non-local and non-covariant, it is important to study the renormalisability of these variablesएण. Similarly we have studied the infra-red problem which, as we argued in $I$, results from the non-trivial asymptotic dynamics which is not properly included in the Green's functions of unphysical asymptotic fields. We have demonstrated in this paper that our fields pass a series of such IR and UV tests with flying colours.

In the soft domain we already knew [8, 9] that an IR finite, on-shell renormalisation of the propagator of our fields was possible at one loop (for both QED and scalar

\footnotetext{
${ }^{9}$ Again, in dimensional regularisation this integral is zero on the mass shell (see footnote 8 ). If we chose to regularise the IR divergences by introducing a small off-shellness $\Delta$, The integrand of (20) would have an extra factor

$$
\frac{2 p^{\prime} \cdot k-k^{2}}{2 p^{\prime} \cdot k-k^{2}+\Delta}+\frac{2 p \cdot k-k^{2}}{2 p \cdot k-k^{2}+\Delta}-\frac{2 p^{\prime} \cdot k-k^{2}}{2 p^{\prime} \cdot k-k^{2}+\Delta} \frac{2 p \cdot k-k^{2}}{2 p \cdot k-k^{2}+\Delta}
$$

${ }^{10}$ We recall the notorious renormalisation problems arising in axial gauges.
} 
QED). This paper has presented efficient methods to demonstrate this and, most importantly, shown that this crucial property can be extended to scattering. Since the dressing depends on the velocity of the charge, in a scattering process we have to associate different dressings to the charges in the distant past and far future. On-shell renormalisation then corresponds to putting each of these fields on shell at the various appropriate points on the mass shell. We have seen that doing this in a self-consistent manner leads to the cancellation of the IR divergences which arise when we go on shell using unphysical asymptotic fields. We stress that this is a very delicate cancellation and that were we to go on shell at a point inappropriate to even one of the dressings then the Green's functions would contain IR divergences and we would not be able to construct $S$-matrix elements!

We were further able to show that the soft divergences, in the on-shell Green's functions of our physical fields and the corresponding $S$-matrix elements, cancel at all orders of perturbation theory. We believe that these results are strong evidence both of the validity of our formalism and of the specific physical interpretation we attach to it.

We also note here in passing that, since for a particle being scattered the appropriate dressings for the incoming and outgoing asymptotic charges are different, there is no gauge such that both dressings in a scattering process vanish (except for the special case of zero momentum transfer when the dressings are of course identical). This is in contrast to the case of the propagator, where there is only one dressing which can be reduced to unity by an appropriate gauge choice. There our formalism can give a physical explanation for some results which were previously just curiosities, such as the IR-finiteness of the on-shell electron propagator in Coulomb gauge if renormalisation was performed at the static point of the mass shell [18].

Since our charged fields are non-local and non-covariant it was not clear in advance what their renormalisation properties would be. In the one-loop propagator, we had previously demonstrated that the UV divergences could be multiplicatively renormalised in this Green's function (via a matrix multiplication for the fermionic theory). This property is highly non-trivial and we have here extended this to study the renormalisation of our physical fields both considered as composite operators and also in vertex functions.

We saw that under renormalisation the dressed electron does not mix with other operators. This property was seen in the simplest possible insertion and is trivially the case for higher Green's functions with one insertion of a physical field.

Proceeding to higher insertions, we considered the propagator and the scattering vertex. We derived a Ward identity linking the scattering vertex for dressed electrons with their propagation. The important physical results which emerged from these studies were that the dressed electron couples to the photon with the usual coupling strength and that the anomalous magnetic moment can be reproduced using physical fields.

There are many natural extensions of the work reported in these two papers and we will conclude this paper by describing some of the more obvious extensions. 
Two clear areas where IR structures directly impede phenomenological progress are collinear divergences, which are a consequence of the masslessness of the (colour charged) gluons, and field theories at finite temperature where soft singularities are much more severe.

The finite temperature case can be attacked in the framework of the abelian theory A first step in the struggle against collinear divergences would be to consider QED with massless matter fields. This is a natural testing ground for QCD, see Sect. 5 of [6] for an initial discussion of this problem.

Confinement makes it obvious that the asymptotic dynamics of QCD can not be neglected. There are two immediate questions here: why do we not see coloured quarks and gluons as physical states and, given this, why can we still describe hadrons so well in terms of constituent quarks?

It is clear that the original fields of the QCD Lagrangian cannot be directly identified with these constituents. The effective quarks of quark models must rather be constructed from the Lagrangian fields. It may be seen [7] that only fields which are locally gauge invariant can have a well-defined colour charge (which is required in any effective quark model).

The primary difference between QCD and QED is that the vector bosons in nonabelian gauge theories carry colour charges and directly interact amongst themselves. This raises a multiplicity of questions, ranging from the structure of glueballs to the construction of pomerons, which last it seems [19] is dominated by glue and not quarks. It is, we note, possible to construct gauge-invariant, dressed gluons in certain domains. The possible phenomenological importance for gauge invariant gluonic operators of dimension two has been stressed many times.

At lowest order in the QCD coupling the anti-screening effect in the beta function is twelve times as large as the opposed screening term. The first non-trivial test of QCD dressings was a study of the interquark potential presented in [10]. Here it was shown that the minimal gauge-invariant extension to QCD of the dressing appropriate to a static charge in QED exactly describes the gluonic configuration which is responsible for asymptotic freedom and anti-screening. This showed that this anti-screening interaction takes place between separately gauge-invariant constituents. This is currently being extended to higher orders in the coupling which will for the first time clarify the interplay between anti-screening and screening beyond the above mentioned lowest order effect.

The electron propagator discussed above is, at one loop, the same as the quark propagator, apart from trivial colour factors. Higher loop investigations will test the non-abelian nature of the dressings appropriate for colour charges. A first test is clearly to use the dressing of [10], generalised to arbitrary velocities, in the two-loop propagator. One would not necessarily expect all the IR divergences to cancel (phasetype structures, see $I$, are missing in the minimal dressing) but, since the primary gluonic effects are evidently included in the dressing, much should indeed cancel. These

\footnotetext{
${ }^{11}$ It is also worth noting that the lower dimensional abelian theory at zero temperature is also plagued by severe soft divergences.
} 


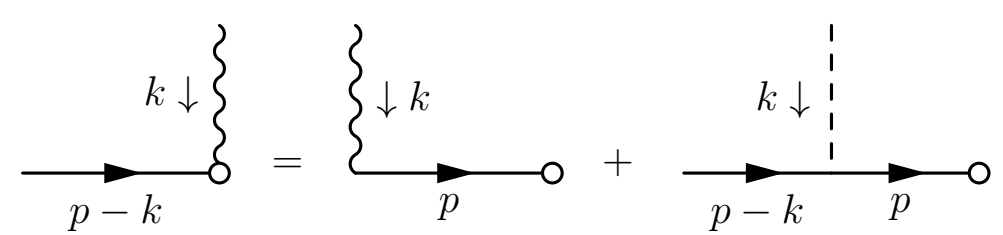

Figure 10: Diagrammatic representation of the identity (77).

studies will open a new window on the interplay between asymptotic freedom and collinear structures.

Acknowledgements: This work was supported by the British Council/Spanish Education Ministry Acciones Integradas grant no. 1801/ HB1997-0141. We thank Robin Horan, Tom Steele, Shogo Tanimura and Izumi Tsutsui for discussions and the organisers of the XIXth UK Theory Institute where some of this work was carried out. EB thanks the HEP group at BNL for their warm hospitality and many interesting comments. He also acknowledges a grant from the Dirección General de Enseñanza Superior e Investigación Científica.

\section{A Factorisation of IR Divergences}

In this appendix we want to demonstrate that the IR divergences associated with what we have called rainbow lines factorise to all orders in perturbation theory. The proof will be diagrammatic.

In a rainbow diagram it appears as though there are no poles associated with the external legs which the rainbow lines are attached to. We recall from Sect. 3 that to extract $S$-matrix elements, we need to find a pole for each particle entering or leaving a scattering process. To be more precise, and again recalling Sect. 3, we note that we are not looking for poles on a diagram by diagram basis, and that they generally come accompanied by IR logarithms of $m^{2}-p^{2}$ which will only cancel in the $S$-matrix elements or in our dressed Green's functions when we go on shell at the right point on the mass shell. However, we do need to extract explicit factors of $1 /(\not p-m)$ (which we will henceforth for simplicity refer to as poles) from the individual diagrams. To obtain these poles from the rainbow diagrams we will below make much use of the general algebraic identities

$$
\begin{aligned}
\frac{1}{\not p-\not k-m} & =\frac{1}{\not p-m}\left[1+\not k \frac{1}{\not p-\not k-m}\right] \\
& =\left[1+\frac{1}{\not p-\not k-m} \not k\right] \frac{1}{\not p-m}
\end{aligned}
$$

The first line of (77) is represented in Fig. 10. Although the vector boson lines are drawn explicitly, they are not written in our identities since they are unaffected by 


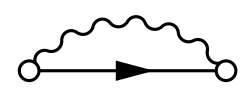

Figure 11: The one-loop propagator rainbow diagram.

our manipulations. Note that we write the photon line in the second term as a dashed line to bring out the fact that there is now an extra factor of $k$ in the numerator. This factor means that the associated integrals over the loop momentum $k$ will now be well defined (see below for examples) and in a diagram where all rainbow lines have been replaced by dashed lines we can safely use standard methods to read off the pole structure. We should stress here that the dressing vertex factors are also unaffected by these manipulations and that the circle should remind the reader that these corrections are still present.

It is significant for what follows, since we want to study both the on-shell IR behaviour of our Green's functions and the UV renormalisation properties, that the terms of the form $\not k /(\not p-\not k-m)$ reduce to -1 both for on-shell $p$ and also in the UV limit of very large $k$. Of course such identities are often used, e.g., to obtain Ward identities. To show the factorisation property we will proceed in this appendix by studying first a one loop and then a two loop rainbow diagram. After these explicit examples, we will consider the general case.

Consider now the simple one loop diagram, Fig. 11. This may be written (up to irrelevant overall factors) as ${ }^{[2]}$

$$
\int \frac{d^{4} k}{(2 \pi)^{4}} \frac{V^{2}}{(V \cdot k)^{2}} \frac{1}{k^{2}} \frac{1}{[\not p-\not k-m]} .
$$

One might be tempted to think that this diagram does not have a pole. However, the non-local nature of the dressing vertices is such that we can indeed extract a pole from this diagram as we now explain. We first note that the integrand is ill-defined: for any $p$, i.e., even off shell, it diverges. The factorisation property which we demonstrate here will allow us to define such terms in a systematic way. Using (77) the integrand may be written as a sum of two terms

$$
\frac{V^{2}}{(V \cdot k)^{2}} \frac{1}{k^{2}} \frac{1}{\not p-m}\left[1+\not k \frac{1}{[\not p-\not k-m]}\right],
$$

the first integral is a massless tadpole and the second is now well-defined. Diagrammatically this is shown in Fig. 12. We stress that the tadpole and explicit pole structures are both clearly visible in the diagrams.

The second diagram has a UV divergence and, when $p$ is on-shell, it also displays an IR divergence, i.e., a logarithm of $m^{2}-p^{2}$. We now discard [3] the massless tadpole

\footnotetext{
${ }^{12}$ Note that although we work in Feynman gauge in this appendix, the extension to a general gauge is trivial.

${ }^{13}$ We stress that the infra-red divergence contained in this tadpole term is an off-shell divergence. At no stage do we throw away IR divergences which first appear on shell.
} 


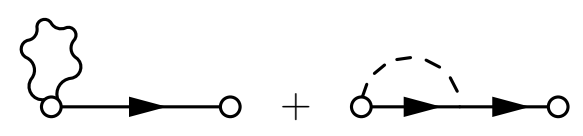

Figure 12: The one-loop rainbow diagram re-expressed using Fig. 10.

since it vanishes in dimensional regularisation and retain the second term which is, as advertised, IR finite off-shell. Furthermore since on-shell (or in the UV region) the second factor in (77) reduces to -1 we see that this loop in those regions does not receive any contribution from the fermion Feynman rules.

It is now apparent that, for our study of the IR structure, this term is just the Feynman rule for the underlying diagram (the free propagator) times a subdiagram which corresponds to the integral $C_{v v}$ (recall Eq. 24) which has been stripped off (factorised) from the initial diagram (recall here Fig. 3). We thus see that, as we previously claimed, we can extract a pole from a rainbow diagram.

To factorise rainbow lines in more general diagrams, one merely needs to make repeated use of such manipulations. Let us further indicate how this works with a two loop example (where we suppress the $1 / 2$ ! symmetry factor).

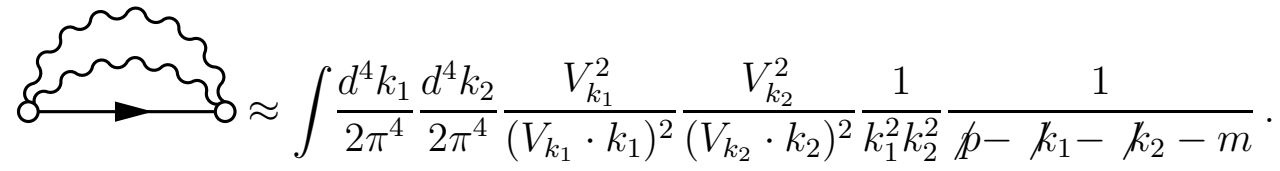

This diagram is ill-defined in both of the $k_{i}$ integrals. Using our basic identities we can write this as

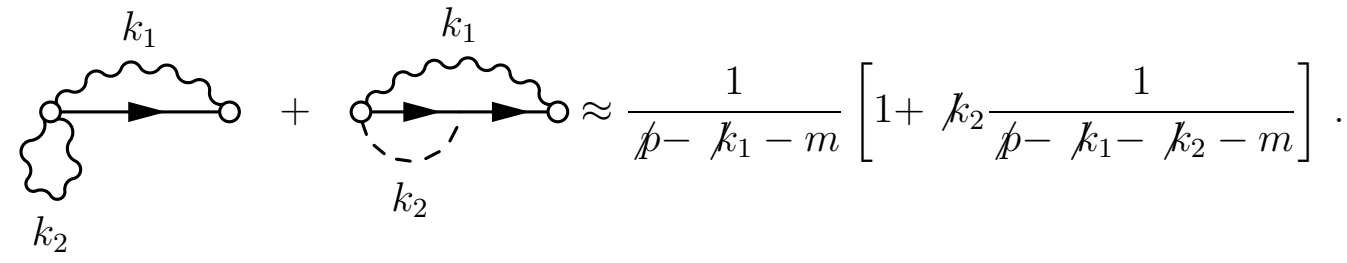

where we have suppressed all factors other than the fermion propagator since it alone is modified by the manipulations that show factorisation. The first term vanishes as it is a massless tadpole. The second structure is IR finite in $k_{2}$ off-shell (but not in $k_{1}$ ) and does not yet have a pole in $1 /(\not p-m)$. However, we can see that but for the remaining rainbow line it would have a pole. We therefore again use (77) to rewrite this second term as

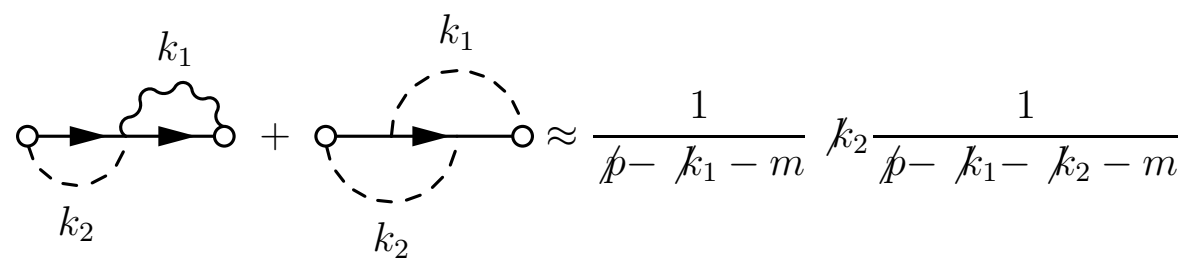




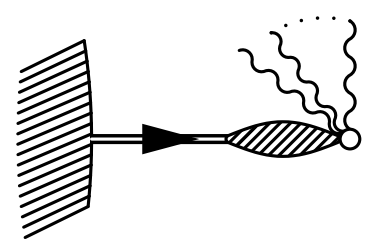

Figure 13: Part of a general diagram with $n$ rainbow lines emitted at an external charged leg.

$$
\times\left[1+\not k_{1} \frac{1}{\not p-\not k_{1}-\not k_{2}-m}\right] \frac{1}{\not p-\not k_{2}-m} .
$$

We see that the second term here is well defined in both loops, but, as the diagram makes apparent, it does not have a pole. (This can be seen algebraically as follows: if we try to extract a pole in $1 /(\not p-m)$ from this figure using (77) the residue vanishes on shell.)

The first term, however, is still ill-defined in one of the loops (the $k_{1}$ rainbow line is not yet a dashed line) and may harbour a pole. Again employing our graphical transformations, Fig. 10, we can recast it as

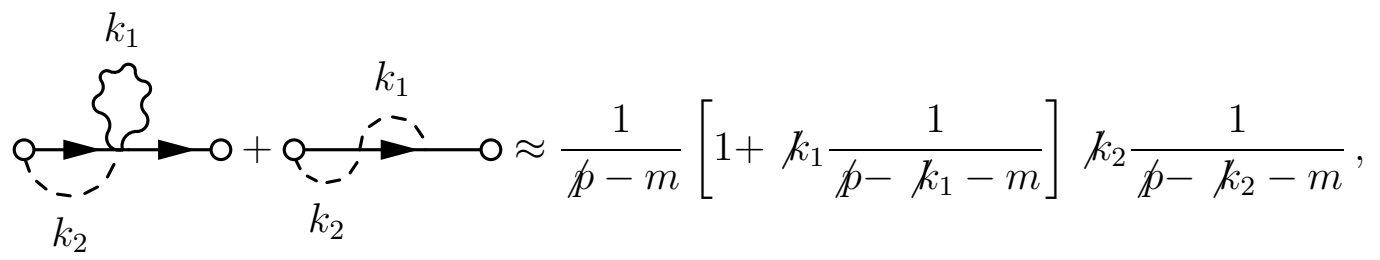

and the first diagram again vanishes since it is a massless tadpole. The second diagram now displays a pole, $1 /(\not p-m)$, but the rainbow lines are now dashed and the two internal loops reduce on-shell to $(-1)^{2}$. Reinstating the factors which we have suppressed we obtain as expected the underlying diagram (here a free propagator) times a factor of $\left(-C_{v v}\right)^{2} / 2$ !. This example brings out all the fundamental ideas of this method.

Consider now a part of a general diagram, Fig. 13, where an external line is connected to one-particle irreducible factors involving the dressing at that line, and to $n$ rainbow lines. These rainbow lines will render the diagram ill-defined. We note that the matter line is written as a double line to denote that the standard QED counterterm diagrams have been included so that the propagator has a pole in $1 /(\not p-m)$ with a logarithmic correction; this is the full renormalised propagator of the Lagrangian matter field (the photon propagator should also be understood as the full renormalised one). This diagram could, for example, be a part of the general scattering diagram of Fig. 6 which, if it were not for the presence of the rainbow lines, would visibly have a pole in each leg.

We now proceed to take the end of one of the rainbow lines to the left through the 1PI factor using the transformations of Fig. 10; at every stage it will either move on to be attached at the next vertex or it will yield a well defined integral. However, these well defined integrals will not have a pole since the line which we are manipulating will extend from an external vertex into the 1PI structure. Therefore, since we only wish to find the poles and their residues, we drop these terms and obtain eventually Fig. 14. 


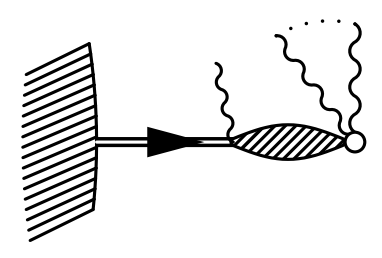

Figure 14: The diagram of Fig. 13 after taking one end of the rainbow line through the visible 1PI blob.

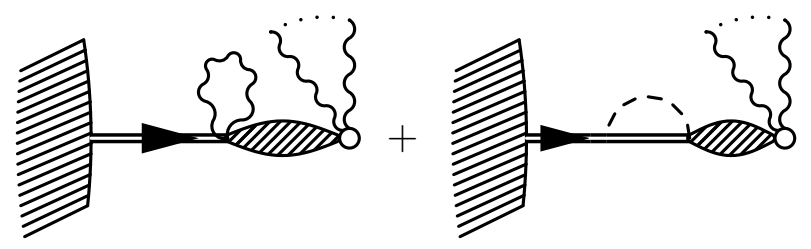

Figure 15: The previous figure when the other end of the rainbow line has been brought through the rest of the diagram.

We now move to the other end of the line which we have been manipulating and again use the identities to bring it towards the right. Once again the diagram which yields a well defined integral will not contain a pole in the matter line which was drawn in Fig. 13, and may be dropped. We repeat this procedure until the second end of the rainbow line reaches the end which we initially manipulated. We then have the diagrams of Fig. 15. The massless tadpole vanishes but we see that the remaining diagram has a pole - up to the as yet unmanipulated rainbow lines. Repeating this procedure for all the rainbow lines will yield Fig. 16. Going on shell each of these loops can be removed and factorisation yields $n$ powers of the form $-C_{v v^{\prime}}$.

Finally we note that if a rainbow line connects two different fermion lines, as might be the case in, say, Møller scattering, then we have to supplement (77) by its photonic equivalent

$$
\frac{1}{(q-k)^{2}}=\frac{1}{q^{2}}\left[1+\frac{2 q \cdot k-k^{2}}{(q-k)^{2}}\right],
$$

which may be expressed diagrammatically in a fashion equivalent to Fig. 10 with the fermion replaced by a hard photon propagator. With this result the above proof may be generalised to any process.

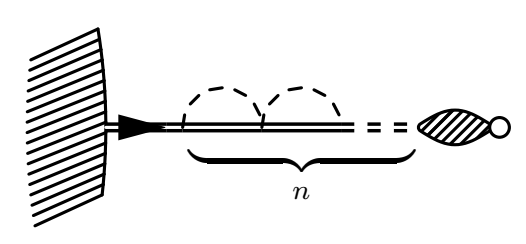

Figure 16: The final result for the diagram after factorising the $n$ rainbow lines. 


\section{B Useful Integrals}

Here we collect some integrals

$$
\begin{aligned}
\int \frac{\mathrm{d}^{2 \omega} k}{(2 \pi)^{2 \omega}} \frac{k_{\mu} k_{\nu}}{\left[(p-k)^{2}-m^{2}\right] k^{2}\left[k^{2}-(k \cdot \eta)^{2}+(k \cdot v)^{2}\right]} & =\frac{i}{32 \pi^{2}} \frac{1}{\hat{\epsilon}}\left[A \eta_{\mu} \eta_{\nu}\right. \\
& \left.+B\left(\eta_{\mu} \eta_{\nu}-g_{\mu \nu}\right)+C v_{\mu} v_{\nu}\right]
\end{aligned}
$$

where

$$
\begin{aligned}
A & =-\chi(\boldsymbol{v}) \\
B & =-\frac{1}{\boldsymbol{v}^{2}}-\frac{1-\boldsymbol{v}^{2}}{2 \boldsymbol{v}^{2}} \chi(\boldsymbol{v}) \\
C & =\frac{3}{\boldsymbol{v}^{4}}+\frac{3-\boldsymbol{v}^{2}}{2 \boldsymbol{v}^{4}} \chi(\boldsymbol{v})
\end{aligned}
$$

Here we have defined

$$
\chi(\boldsymbol{v})=\frac{1}{|\boldsymbol{v}|} \log \frac{1+|\boldsymbol{v}|}{1-|\boldsymbol{v}|}
$$

We also need the integrals

$$
\begin{aligned}
\int \frac{\mathrm{d}^{2 \omega} k}{(2 \pi)^{2 \omega}} \frac{k_{\mu} k_{\nu}}{\left[(p-k)^{2}-m^{2}\right]\left[k^{2}-(k \cdot \eta)^{2}+(k \cdot v)^{2}\right]^{2}} & =\frac{i}{32 \pi^{2}} \frac{1}{\hat{\epsilon}}\left[A \eta_{\mu} \eta_{\nu}\right. \\
& \left.+B\left(\eta_{\mu} \eta_{\nu}-g_{\mu \nu}\right)+C v_{\mu} v_{\nu}\right]
\end{aligned}
$$

where

$$
\begin{aligned}
A & =-\frac{2}{1-\boldsymbol{v}^{2}}+\chi(\boldsymbol{v}), \\
B & =\frac{1}{\boldsymbol{v}^{2}}+\frac{1+\boldsymbol{v}^{2}}{2 \boldsymbol{v}^{2}} \chi(\boldsymbol{v}), \\
C & =-\frac{3-\boldsymbol{v}^{2}}{\boldsymbol{v}^{4}\left(1-\boldsymbol{v}^{2}\right)}-\frac{3+\boldsymbol{v}^{2}}{2 \boldsymbol{v}^{4}} \chi(\boldsymbol{v}) .
\end{aligned}
$$

Finally

$$
\begin{array}{r}
\int \frac{\mathrm{d}^{2 \omega} k}{(2 \pi)^{2 \omega}} \quad \frac{k_{\mu} k_{\nu}}{\left[(p-k)^{2}-m^{2}\right]\left[k^{2}-(k \cdot \eta)^{2}\right]\left[k^{2}-(k \cdot \eta)^{2}+(k \cdot v)^{2}\right]} \\
=\frac{i}{32 \pi^{2}} \frac{1}{\hat{\epsilon}}\left[A \eta_{\mu} \eta_{\nu}+B\left(\eta_{\mu} \eta_{\nu}-g_{\mu \nu}\right)+C v_{\mu} v_{\nu}\right]
\end{array}
$$

where

$$
\begin{aligned}
A & =2 \chi(\boldsymbol{v}) \\
B & =-\frac{2}{\boldsymbol{v}^{2}}-\frac{1-\boldsymbol{v}^{2}}{\boldsymbol{v}^{2}} \chi(\boldsymbol{v}) \\
C & =\frac{6}{\boldsymbol{v}^{4}}+\frac{3-\boldsymbol{v}^{2}}{\boldsymbol{v}^{4}} \chi(\boldsymbol{v}) .
\end{aligned}
$$

This result can be easily obtained by writing (96) as

$$
\int_{0}^{1} \mathrm{~d} x \int \frac{\mathrm{d}^{2 \omega} k}{(2 \pi)^{2 \omega}} \frac{k_{\mu} k_{\nu}}{\left[(p-k)^{2}-m^{2}\right]\left[k^{2}-(k \cdot \eta)^{2}+x(k \cdot v)^{2}\right]^{2}},
$$

and using (91), (92), (93) and (94). 


\section{Gauge invariance and a Ward Identity}

In this appendix we prove the Ward identity that was used in Sect. 1 , i.e., $Z(v, v)=1$. We will also show that, as a result of gauge invariance, $q_{\mu} V_{v v^{\prime}}^{\mu}\left(p, p^{\prime}\right)=0$.

Let us start with the identity $q_{\mu} V_{v v^{\prime}}^{\mu}\left(p, p^{\prime}\right)=0$. This can be most easily demonstrated by considering BRST invariance in the path integral formalism. If $\bar{c}(x)$ is the anti-ghost field, we can write

$$
0=\delta_{\mathrm{BRS}}\left\langle 0\left|\mathrm{~T} \psi_{v^{\prime}}(y) \bar{\psi}_{v}(0) \bar{c}(x)\right| 0\right\rangle \propto \frac{\partial}{\partial x^{\mu}}\left\langle 0\left|\mathrm{~T} \psi_{v^{\prime}}(y) \bar{\psi}_{v}(0) A^{\mu}(x)\right| 0\right\rangle
$$

where we recall that in the path integral formalism, derivatives are understood to act outside the time ordering. Taking the Fourier transform of this equation, one obtains

$$
0=q_{\mu}\left\langle\psi_{v^{\prime}}\left(p^{\prime}\right) \bar{\psi}_{v}(p) A^{\mu}(q)\right\rangle=i q^{\mu} D_{\mu \nu}(q) i e V_{v v^{\prime}}^{\nu}\left(p, p^{\prime}\right)
$$

where we have used the definition (58). Note that by momentum conservation, $q=$ $p^{\prime}-p$. Further recall that both the free photon propagator, $D_{\mu \nu}^{(0)}(q)$, and the full one $D_{\mu \nu}(q)$, satisfy in an arbitrary Lorentz gauge

$$
q^{\mu} D_{\mu \nu}^{(0)}(q)=q^{\mu} D_{\mu \nu}(q)=-\xi \frac{q_{\nu}}{q^{2}},
$$

which may be expressed as saying that the longitudinal part of the photon propagator is not renormalised. From (101) and (102), and for a non-zero gauge parameter $\xi$, we readily see that

$$
q_{\mu} V_{v v^{\prime}}^{\mu}\left(p, p^{\prime}\right)=0
$$

We will return to this identity, and present an alternative proof of it using the canonical formalism, at the end of this appendix.

We now want to derive the Ward identity, (61). Let us consider Eq. 58 for $v^{\prime}=v$. Looking at the diagrams of Fig. 17, we see that we can recast the right hand side as (we drop the B superscript for simplicity)

$$
i e V_{v v}^{\nu}\left(p, p^{\prime}\right)=i e\left\langle\psi_{v}\left(p^{\prime}\right) \bar{\psi}_{v}(p) J^{\nu}(q)\right\rangle^{1 \mathrm{PhI}}-i S_{v}\left(p^{\prime}\right) \frac{e V^{\nu}}{V \cdot q}+\frac{e V^{\nu}}{V \cdot q} i S_{v}(p)
$$

where $J^{\nu}$ is the (conserved) electromagnetic current, $J^{\nu}(x)=\bar{\psi}(x) \gamma^{\nu} \psi(x)$. The superscript "1PhI" means one photon irreducible. A diagram is called $1 \mathrm{PhI}$ if it cannot be split into two parts by cutting a photon line.

Recalling again (102), we obtain from (104) and (58)

$$
q_{\nu} V_{v v}^{\nu}\left(p, p^{\prime}\right)=q_{\nu}\left\langle\psi_{v}\left(p^{\prime}\right) \bar{\psi}_{v}(p) J^{\nu}(q)\right\rangle-S_{v}\left(p^{\prime}\right)+S_{v}(p) .
$$

Therefore, Eq. 103 trivially implies

$$
q_{\nu}\left\langle\psi_{v}\left(p^{\prime}\right) \bar{\psi}_{v}(p) J^{\nu}(q)\right\rangle=S_{v}\left(p^{\prime}\right)-S_{v}(p) .
$$

We now define

$$
i e \mathfrak{M}_{v v^{\prime}}^{\mu}\left(p, p^{\prime}\right)=\left[i S_{v^{\prime}}\left(p^{\prime}\right)\right]^{-1} i e\left\langle\psi_{v^{\prime}}\left(p^{\prime}\right) \bar{\psi}_{v}(p) J^{\mu}(q)\right\rangle^{1 \mathrm{PhI}}\left[i S_{v}(p)\right]^{-1} .
$$




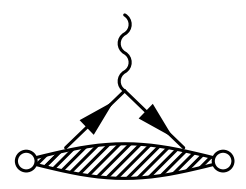

(a)

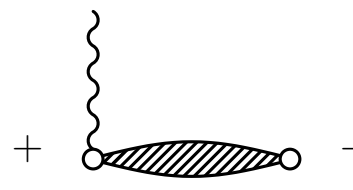

(b)

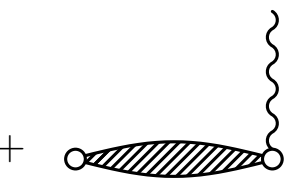

(c)

Figure 17: Graphical representation of (104). The blobs represent irreducible contributions for the photon and all contributions (i.e., including reducible ones) for the fermions. The photon lines are amputated.

When computing $S$-matrix elements (see $I$ ), only diagrams with a pole for each external leg (here one in $p$ and one in $p^{\prime}$ ) are relevant, since, in the LSZ formalism we will have to amputate such external legs. It is thus clear that one can construct the $S$-matrix element from (107) for the scattering process since all the diagrams with two poles are contained in the Green's function, $\left\langle\psi_{v^{\prime}}\left(p^{\prime}\right) \bar{\psi}_{v}(p) J^{\nu}(q)\right\rangle$. These diagrams are contained in Fig. 17a; we stress that Fig.'s 17b and 17c do not contain two poles and cannot contribute to the $S$-matrix.

We need a renormalisation condition and, in the on-shell scheme, we impose on the irreducible photon-dressed electron vertex

$$
\bar{u}\left(p, s^{\prime}\right) \mathfrak{M}_{v v}^{\mathrm{R} \mu}(p, p) u(p, s)=\bar{u}\left(p, s^{\prime}\right) \gamma^{\mu} u(p, s)
$$

in analogy to the well known condition

$$
\bar{u}\left(p, s^{\prime}\right) \Gamma^{\mathrm{R} \mu}(p, p) u(p, s)=\bar{u}\left(p, s^{\prime}\right) \gamma^{\mu} u(p, s)
$$

for the standard QED vertex. Eq. 108 determines what we mean by the physical charge, i.e., the charge that would be experimentally measured at low momentum transfer.

It suffices, of course, to prove that $Z(v, v)=1$ in the on-shell scheme since the UV divergent part of the renormalisation constants is the same in any scheme, this will also prove that $Z(v, v)=1$ in, say, the MS or $\overline{\mathrm{MS}}$ schemes.

Note that from (107) and (106) we have

$$
q_{\mu} \mathfrak{M}_{v v}^{\mu}\left(p, p^{\prime}\right)=\left[S_{v}\left(p^{\prime}\right)\right]^{-1}-\left[S_{v}(p)\right]^{-1}
$$

where we should point out that the dressing parameters are chosen to be identical. In (106) we can write the expectation value as either $1 \mathrm{PhI}$ or not, since dotting $q$ into the full photon propagator kills everything except for the tree level propagator. Multiplying on both sides with renormalisation constants and sandwiching between spinors yields

$$
\bar{u}\left(p, s^{\prime}\right) \sqrt{\mathbf{Z}_{v}} q_{\mu} \mathfrak{M}_{v v}^{\mu}\left(p, p^{\prime}\right) \sqrt{\mathbf{Z}_{v}} u(p, s)=\bar{u}\left(p, s^{\prime}\right) \sqrt{\overline{\mathbf{Z}_{v}}}\left(\left[S_{v}\left(p^{\prime}\right)\right]^{-1}-\left[S_{v}(p)\right]^{-1}\right) \sqrt{\mathbf{Z}_{v}} u(p, s) .
$$

Note now that from the definition of $\mathfrak{M}_{v v^{\prime}}^{\mu}\left(p, p^{\prime}\right)$ one has

$$
\mathfrak{M}_{v v}^{\mu}\left(p, p^{\prime}\right)=Z(v, v)\left(\sqrt{\overline{\mathbf{Z}_{v}}}\right)^{-1} \mathfrak{M}_{v v}^{\mathrm{R} \mu}\left(p, p^{\prime}\right)\left(\sqrt{\mathbf{Z}_{v}}\right)^{-1}
$$


To obtain this result we have employed (60) and we further note that the different terms on the right hand side of (104) must all renormalise in the same way since they have completely different pole structures. Using this and the definition of the renormalisation constants (14) for $S_{v}(p)$, we have

$$
Z(v, v) \bar{u}\left(p, s^{\prime}\right) q_{\mu} \mathfrak{M}_{v v}^{\mathrm{R} \mu}\left(p, p^{\prime}\right) u(p, s)=\bar{u}\left(p, s^{\prime}\right)\left(\left[S_{v}^{R}\left(p^{\prime}\right)\right]^{-1}-\left[S_{v}^{R}(p)\right]^{-1}\right) u(p, s) .
$$

The last term on the right hand side vanishes because of the spinor and we can similarly reexpress the first term to obtain

$$
Z(v, v) \bar{u}\left(p, s^{\prime}\right) q_{\mu} \mathfrak{M}_{v v}^{\mathrm{R} \mu}\left(p, p^{\prime}\right) u(p, s)=\bar{u}\left(p, s^{\prime}\right) \dot{q} u(p, s) .
$$

From our renormalisation condition, the desired result, $Z(v, v)=1$, follows.

We conclude this appendix with an alternative derivation of (106), and hence (103) for $v^{\prime}=v$ using the canonical formalism. If we notice that the left hand side of the former is the Fourier transform of

$$
\frac{\partial}{\partial x^{\nu}}\left\langle 0\left|\mathrm{~T} \psi_{v}(y) \bar{\psi}_{v}(0) J^{\nu}(x)\right| 0\right\rangle,
$$

then upon taking the derivative inside the time ordered product and recalling the conservation of electric charge, $\partial \cdot J=0$, and the fundamental equal-time (anti-)commutation relations, it follows that

$$
\frac{\partial}{\partial x^{\nu}}\left\langle 0\left|\mathrm{~T} \psi_{v}(y) \bar{\psi}_{v}(0) J^{\nu}(x)\right| 0\right\rangle=\left[\delta^{4}(x)-\delta^{4}(x-y)\right]\left\langle 0\left|\mathrm{~T} \psi_{v}(y) \bar{\psi}_{v}(0)\right| 0\right\rangle .
$$

In momentum space this identity can be recast as (106). Substituting into (105) we finally obtain

$$
q_{\nu} V_{v v}^{\nu}\left(p, p^{\prime}\right)=0 .
$$

The generalisation of this proof to $v^{\prime} \neq v$ is straightforward.

We also note that for identical dressings $\left(v=v^{\prime}\right)$ the Ward identity (106) can be understood as the usual identity in a particular (dressing) gauge.

\section{References}

[1] J. Dollard, J. Math. Phys. 5, 729 (1964).

[2] V. Chung, Phys. Rev. B 140, 1110 (1965).

[3] P. P. Kulish and L. D. Faddeev, Theor. Math. Phys. 4, 745 (1970).

[4] E. Bagan, M. Lavelle, and D. McMullan, (1999), Charges from Dressed Matter: Construction, BNL-HET-99/18, PLY-MS-99-23, submitted for publication.

[5] E. Bagan, M. Lavelle, and D. McMullan, Phys. Rev. D57, 4521 (1998), hepth/9712080. 
[6] R. Horan, M. Lavelle, and D. McMullan, Pramana J. Phys. 51, 317 (1998), hepth/9810089, Erratum-ibid, 51 (1998) 235.

[7] M. Lavelle and D. McMullan, Phys. Rept. 279, 1 (1997), hep-ph/9509344.

[8] E. Bagan, B. Fiol, M. Lavelle, and D. McMullan, Mod. Phys. Lett. A12, 1815 (1997), hep-ph/9706515, Erratum-ibid, A12 (1997) 2317.

[9] E. Bagan, M. Lavelle, and D. McMullan, Phys. Rev. D56, 3732 (1997), hepth/9602083.

[10] M. Lavelle and D. McMullan, Phys. Lett. B436, 339 (1998), hep-th/9805013.

[11] S. Weinberg, The Quantum Theory of Fields, Vol. I (Cambridge University Press, Cambridge, 1995).

[12] R. Jackiw and L. Soloviev, Phys. Rev. 27, 1485 (1968).

[13] J. C. Collins, Renormalization. An Introduction to Renormalization, the Renormalization Group, and the Operator Product Expansion (Cambridge University Press, Cambridge, 1984).

[14] N. Isgur and M. B. Wise, Phys. Lett. B237, 527 (1990).

[15] N. Isgur and M. B. Wise, Phys. Lett. B232, 113 (1989).

[16] V. S. Dotsenko and S. N. Vergeles, Nucl. Phys. B169, 527 (1980).

[17] R. A. Brandt, F. Neri, and M. Sato, Phys. Rev. D24, 879 (1981).

[18] T. W. B. Kibble, Phys. Rev. 173, 1527 (1968).

[19] H1, C. Adloff et al., Z. Phys. C76, 613 (1997), hep-ex/9708016. 